\title{
$B$ decay anomalies and dark matter from vectorlike confinement
}

\author{
James M. Cline \\ Niels Bohr International Academy and Discovery Center, Niels Bohr Institute, University of Copenhagen, \\ Blegdamsvej 17, DK-2100 Copenhagen, Denmark \\ and McGill University, Department of Physics, \\ 3600 University Street, Montréal, Quebec H3A2T8, Canada
}

(Received 18 October 2017; published 25 January 2018)

\begin{abstract}
Lepton flavor universality violating $B \rightarrow K \ell \ell$ and $K^{*} \ell \ell$ decays tentatively observed by LHCb can be explained by leptoquark exchange. We explore a simple model for the $B$ anomalies with a composite leptoquark from new strong dynamics at the $\mathrm{TeV}$ scale, a confining $\mathrm{SU}\left(N_{H C}\right)$ hypercolor interaction. The new matter fields, fundamentals under $\mathrm{SU}\left(N_{H C}\right)$, are heavy vectorlike fermions $\Psi, S$, and an inert scalar doublet $\phi$. $\Psi$ is colored under QCD while $S$ is neutral, and the hyperbaryon $S^{N}$ is an asymmetric dark matter candidate. The model is tightly constrained by meson-antimeson oscillations, lepton flavor violation, and LHC searches for resonant production of the exotic bound states. The dark matter may be detectable through its magnetic dipole moment. If $m_{S}$ is sufficiently small, composite leptoquarks and heavy lepton partners can be pair-produced at an observable level at LHC.
\end{abstract}

DOI: 10.1103/PhysRevD.97.015013

\section{INTRODUCTION}

While the Standard Model (SM) continues to accurately describe particle interactions at the shortest probed distance scales, there is an encouraging hint of new physics from decays of neutral $B$ mesons to $K$ or $K^{*}$ and charged leptons. The LHCb collaboration measures the ratios of branching ratios $[1,2]$

$$
R_{K}^{(*)}=\frac{B\left(B \rightarrow K^{(*)} \mu^{+} \mu^{-}\right)}{B\left(B \rightarrow K^{(*)} e^{+} e^{-}\right)}
$$

to be (20-30)\% below the SM prediction for both $K$ and $K^{*}$ final states. Uncertainties from the hadronic matrix elements cancel in the ratios, greatly reducing the theoretical errors and making these ratios particularly interesting probes of new physics that could violate lepton flavor universality. Although the discrepancies between theory and experiment in the individual measurements are below $3 \sigma$, combining them increases the significance to $4 \sigma$ [3-13]. This conclusion remains true when other observables are included in the fit, like the branching ratio for $B_{s} \rightarrow \phi \mu^{+} \mu^{-}$, which also has a deficit, and angular decay distributions that are subject to hadronic uncertainties.

Model-independent fits to the data show that the addition of the single effective operator

Published by the American Physical Society under the terms of the Creative Commons Attribution 4.0 International license. Further distribution of this work must maintain attribution to the author(s) and the published article's title, journal citation, and DOI. Funded by SCOAP ${ }^{3}$.

$$
\mathcal{O}_{b_{L} \mu_{L}}=\frac{c}{\Lambda^{2}}\left(\bar{s}_{L} \gamma_{\alpha} b_{L}\right)\left(\bar{\mu}_{L} \gamma^{\alpha} \mu_{L}\right)
$$

to the effective Hamiltonian is sufficient to give a good fit to the observations, with [4]

$$
\frac{c}{\Lambda^{2}}=\frac{1.0 \times 10^{-3}}{\mathrm{TeV}^{2}} \text {. }
$$

The form (2) arises in the standard model from $W$ exchange at one loop, and can be realized in models of new physics loop effects [14-20]. It could also be induced by exchange of a heavy $Z^{\prime}$ vector boson, inspiring the construction of many models of this kind [21-54]. By Fierz rearrangement, (2) takes the form suggestive of vector leptoquark exchange. Global analyses identify two possible vector leptoquarks $U_{1}$ and $U_{3}$ and one scalar $S_{1}$ as viable candidates, in the notation of Ref. [55] where the subscript denotes the $\mathrm{SU}(2)_{L}$ representation. Models involving leptoquark exchange have been studied in Refs. [15,56-87].

In the present work we offer a simple, UV complete model in which the vector $U_{1}$ leptoquark arises from confining dynamics of an $\mathrm{SU}\left(N_{H C}\right)$ gauge theory, referred to as hypercolor. It is an example of vectorlike confinement [88] in which the constituent fermions have bare masses that can be freely chosen. Our proposal differs from previous models for $R_{K^{(*)}}$ with composite leptoquarks [57,71,78,81] where the constituent masses were assumed to be much lighter than the confinement scale $\Lambda_{H C}$. In that case, approximate chiral symmetry is important, and the lightest bound states are pseudo-Nambu-Goldstone bosons (pNGBs).

Here we instead assume that the constituent masses $M$ are larger than $\Lambda_{H C}$ (but not too much larger) so that chiral 
dynamics plays no role, yet the system is not quirky [89] as occurs if $\Lambda_{H C} \ll M$. One advantage of this choice is that the strong dynamics is somewhat more calculable, via a nonrelativistic potential model that works reasonably well for QCD-onium states like $J / \psi$ and $\Upsilon{ }^{1}$. Moreover, collider constraints on production of the new bound states are under better control in this regime, where resonant production is suppressed by the wave function of the constituents, and pair production is limited by the large bound state masses. We also thereby avoid complications associated with composite Higgs scenarios.

Another distinctive feature of our model is that it provides a dark matter (DM) candidate, which is intimately linked to the $B$ decay phenomenology. In particular, one of the hyperquarks (denoted by $S$ ) is a singlet under the SM gauge group. It is a constituent of the composite leptoquark, and the baryonlike $S^{N_{H C}}$ bound state is the dark matter. Exceptionally, $S$ can be lighter than $\Lambda_{H C}$ without entailing a pNGB, since the approximate $\mathrm{U}(1)_{A}$ flavor symmetry associated with $S$ is anomalous. Since it carries a conserved number, this is an asymmetric DM particle, which can attain the observed relic density through generation of an asymmetry by a genesis mechanism that we do not specify. It will be shown that the symmetric component is much smaller than the observed DM relic density.

A further difference relative to previous models of composite leptoquarks is that we invoke a scalar hyperquark $\phi$, a doublet under $\mathrm{SU}(2)_{L}$. It is motivated by the need to couple the new physics to left-handed quarks and leptons [Eq. (2)]. Previous models achieved this by taking the fermionic hyperquarks to be doublets, but it is more straightforward to have a dark matter candidate if this is avoided, and the massless limit of $S$ can be safely taken without introducing new relatively light scalars that would be easily produced in colliders. In our model both fermionic hyperquarks, $S$ and $\Psi$, are isosinglets, while $\Psi$ carries color. The $S \bar{\Psi}$ bound state is the leptoquark.

After defining the model in Sec. II and reducing it to an effective theory suitable for addressing the $B$ decay anomalies in Sec. III, we identify (without fully delineating) some regions of parameter space that are consistent with constraints on flavor changing neutral currents (FCNCs) in Sec. IV, dark matter direct detection (Sec. V), and collider searches (Sec. VI). The examples we focus on have potential for associated new signals in all of these categories of observables. A summary and conclusions are given. Appendixes provide details of the potential model for estimating bound state properties, the formalism for computing dipole moments from partial compositeness of the light fermions, and the magnetic dipole moment of the dark matter constituent fermion.

\footnotetext{
${ }^{1}$ Nevertheless, we don't expect better than $~ 30 \%$ accuracy from this approach, and will indicate any determinations that are

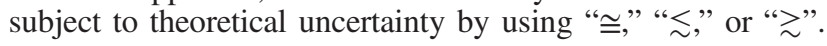

\section{MODEL}

Our model introduces three matter fields that are fundamentals of hypercolor (HC): a Dirac fermionic DM particle $S$, a vectorlike fermion $\Psi$ that carries SM color, and a scalar $\phi$ that is an $\mathrm{SU}(2)_{L}$ doublet. The quantum numbers are shown in Table I. Gauge symmetry allows these fields to couple to left-handed SM quarks and leptons only through the interactions

$$
\mathcal{L}=\tilde{\lambda}_{i} \bar{Q}_{i, a} \phi_{A}^{a} \Psi^{A}+\lambda_{i} \bar{S}_{A} \phi_{a}^{* A} L_{i}^{a}
$$

where $a(A)$ is the $\mathrm{SU}(2)_{L}\left(\mathrm{SU}(N)_{H C}\right)$ index and $i$ is the flavor index. These interactions explicitly break the approximate flavor symmetries of the standard model (SM), and so one can anticipate that the matching of the observed $b \rightarrow s \mu^{+} \mu^{-}$FCNC will give rise to other FCNC observables. The observed $B$ decay anomaly is mediated by tree-level exchange of a composite leptoquark $\Phi=\Psi \bar{S}$, while the other kinds of bound states $\Psi \bar{\Psi}$ and $S \bar{S}$ give analogous contributions to neutral meson mixing and flavor-violating decays of charged leptons, depicted in Fig. 1.

To fully specify the model, we must define the flavor basis of the SM fields in (4). For simplicity we assume that the mass matrices of the charged leptons and down-type quarks are already diagonal in this basis, so that all the mixing giving rise to the CKM matrix is due to diagonalizing the up-type quark masses. Then going to the mass eigenbasis of the quarks,

$$
\tilde{\lambda}_{i} \bar{Q}_{i} \rightarrow \tilde{\lambda}_{j}\left(\bar{u}_{L, i} V_{i j}, \bar{d}_{L, j}\right) \equiv\left(\tilde{\lambda}_{i}^{\prime} \bar{u}_{i}, \tilde{\lambda}_{i} \bar{d}_{i}\right)
$$

Thus, the couplings to up-type quarks are given by $\tilde{\lambda}_{i}^{\prime}=V_{i j} \tilde{\lambda}_{j}$.

It is worth noting that the individual lepton flavor symmetry for generation $i$ becomes exact in the limit $\lambda_{i} \rightarrow 0$ : these couplings are only multiplicatively renormalized, up to neutrino mass insertions. Therefore, if we only wish to explain lepton flavor universality violation in the muon sector, it is technically natural to set the couplings $\lambda_{1}, \lambda_{3} \rightarrow 0$.

If $S$ is lighter than $\phi$ and $\Psi$, the hyperbaryon bound state $\Sigma=S^{N}$ is a dark matter candidate. Its stability is protected by the accidental $Z_{2}$ symmetry under which all the new matter fields are odd. It is a consequence of the $\mathrm{SU}\left(N_{H C}\right)$ gauge symmetry, analogous to baryon number conservation

TABLE I. Quantum numbers of new physics particles.

\begin{tabular}{lcccccc}
\hline \hline & $\mathrm{SU}(3)$ & $\mathrm{SU}(2)_{L}$ & $\mathrm{U}(1)_{y}$ & $\mathrm{U}(1)_{\mathrm{em}}$ & $\mathrm{SU}(N)_{H C}$ & $Z_{2}$ \\
\hline$\Psi$ & 3 & 1 & $2 / 3$ & $2 / 3$ & $N$ & -1 \\
$S$ & 1 & 1 & 0 & 0 & $N$ & -1 \\
$\phi$ & 1 & 2 & $-1 / 2$ & $(0,-1)$ & $\bar{N}$ & -1 \\
\hline \hline
\end{tabular}




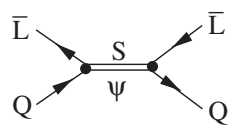

(a)

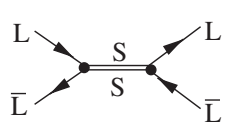

(b)

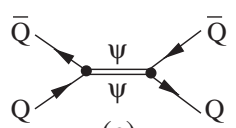

(c)
FIG. 1. Tree-level contributions to flavor-violating processes from exchange of composite vector bosons.

in the SM. Moreover, the fields $\Psi$ and $S$ can consistently be assigned normal baryon and lepton numbers, respectively.

\section{LOW-ENERGY EFFECTIVE THEORY}

The $\bar{\Psi} S$ bound states of our model have the quantum numbers of leptoquarks and contribute to the decays $B \rightarrow K^{(*)} \mu^{+} \mu^{-}$. The leptoquarks can be either pseudoscalar $\Pi$ or vector $\rho_{\mu}$, with decay constants

$$
\begin{aligned}
\left\langle 0\left|\left(\bar{S} \gamma^{\mu} \gamma_{5} \Psi\right)\right| \Pi\right\rangle & =f_{\Pi} p_{\Pi}^{\mu}, \\
\left\langle 0\left|\left(\bar{S} \gamma^{\mu} \Psi\right)\right| \rho_{\lambda}\right\rangle & =f_{\rho} m_{\rho} \epsilon_{\lambda}^{\mu},
\end{aligned}
$$

where $\lambda$ labels the helicity state of the vector. These currents couple to the SM fields via $\bar{Q} \gamma_{\mu} L$ and so the interactions of the pseudoscalar are suppressed by small quark and lepton masses through the equations of motion due to the momentum factor $p_{\Pi}^{\mu}=p_{q}^{\mu}+p_{l}^{\mu}$. This can also be understood in terms of the helicity suppression of the amplitude for pseudoscalar decay to approximately chiral states in analogy to charged pion decay. Hence, we are interested in the vector leptoquark for explaining the $B$ decay anomalies.

We seek an effective description of the leptoquark interaction with the SM fields,

$$
g_{\rho}^{i j} \rho_{\mu}\left(\bar{Q}_{i} \gamma^{\mu} L_{j}\right)
$$

The coupling $g_{\rho}^{i j}$ can be determined by matching the decay rate for $\rho_{\mu} \rightarrow Q_{i} \bar{L}_{j}$ in the effective theory and the underlying model. In the effective theory, the rate is

$$
\Gamma\left(\rho_{\mu} \rightarrow Q_{i} \bar{L}_{j}\right)=\frac{\left|g_{\rho}^{i j}\right|^{2}}{24 \pi} m_{\rho},
$$

neglecting the quark and lepton masses. In the UV theory, this rate can be computed as $\Gamma=\sigma v_{\text {rel }}|\psi(0)|^{2}$, where $\psi$ is the wave function for the $\Psi \bar{S}$ bound state and $\sigma$ is the cross section for $\Psi \bar{S} \rightarrow Q_{i} \bar{L}_{j}$ annihilation,

$$
\sigma v_{\text {rel }}=N_{H C} \frac{\left|\tilde{\lambda}_{i}^{2} \lambda_{j}^{2}\right|\left(m_{S}+m_{\Psi}\right)^{2}}{96 \pi\left(m_{S} m_{\Psi}+m_{\phi}^{2}\right)^{2}},
$$

ignoring the initial velocities of the bound particles, and assuming only the spin- 1 configuration of $S$ and $\Psi$ contributes in the sum over spins. This gives

$$
g_{\rho}^{i j}=\left(\frac{N_{H C}}{4 m_{\rho}}\right)^{1 / 2} \frac{\tilde{\lambda}_{i} \lambda_{j}^{*}\left(m_{S}+m_{\Psi}\right)}{\left(m_{S} m_{\Psi}+m_{\phi}^{2}\right)} \psi(0) .
$$

Once the effective interaction (7) is specified, we can integrate out the leptoquark to generate the dimension- 6 operator shown in Fig. 1(a),

$$
\delta \mathcal{L}=-\frac{g_{i j} g_{i^{\prime} j^{\prime}}^{*}}{m_{\rho}^{2}}\left(\bar{Q}_{i}^{a} \gamma^{\mu} Q_{i^{\prime}, b}\right)\left(\bar{L}_{j^{\prime}}^{b} \gamma^{\mu} L_{j, a}\right),
$$

after Fierz transforming [90], where $a, b$ are $\mathrm{SU}(2)_{L}$ indices. This contribution must interfere destructively with the SM contribution to $b \rightarrow s \mu^{+} \mu^{-}$, requiring $g_{32}^{*} g_{22}$ to be approximately real and positive.

In addition to the composite leptoquarks, there are composite $\bar{\Psi} \Psi \equiv \rho_{\Psi}$ and $\bar{S} S \equiv \rho_{S}$ vector bosons, whose exchanges are shown in Figs. 1(b) and 1(c). The ensuing operators are

$$
\begin{aligned}
\delta \mathcal{L}= & -\frac{h_{i j} h_{i^{\prime} j^{\prime}}^{*}}{2 m_{\rho_{S}}^{2}}\left(\bar{L}_{i}^{a} \gamma^{\mu} L_{i^{\prime}, b}\right)\left(\bar{L}_{j^{\prime}}^{b} \gamma^{\mu} L_{j, a}\right) \\
& -\frac{k_{i j} k_{i^{\prime} j^{\prime}}^{*}}{2 m_{\rho_{\Psi}}^{2}}\left(\bar{Q}_{i}^{a} \gamma^{\mu} Q_{i^{\prime}, b}\right)\left(\bar{Q}_{j^{\prime}}^{b} \gamma^{\mu} Q_{j, a}\right),
\end{aligned}
$$

where $h_{i j}$ and $k_{i j}$ are determined analogously to (10), with $\tilde{\lambda}_{i} \lambda_{j}^{*} \rightarrow \lambda_{i} \lambda_{j}^{*}$ and $\tilde{\lambda}_{i} \tilde{\lambda}_{j}^{*}$, respectively.

\section{FLAVOR CONSTRAINTS}

The fit of Ref. [4] implies that the $R_{K^{(*)}}$ anomalies can be explained by taking

$$
\frac{g_{22} g_{32}^{*}}{m_{\rho}^{2}}=\frac{1 \times 10^{-3}}{\mathrm{TeV}^{2}} .
$$

To translate this into a constraint on parameters of the model, we must determine $\psi(0)$ and the bound state mass. For this purpose we use a potential model that is described in Appendix A. To simplify this preliminary analysis, we assume that $m_{\Psi} \cong m_{\Phi}$. ${ }^{2}$ We consider two possibilities for the dark constituent mass, $\Lambda_{H C} \lesssim m_{S} \lesssim m_{\phi} \equiv M$ and $m_{S} \ll \Lambda_{H C} \lesssim m_{\phi}$, such that $S$ is respectively nonrelativistic or relativistic within the bound state. Two versions of the potential model are given to treat these cases. We avoid the quirky regime where $\Lambda_{H C} \ll M$ [89] since collider constraints are expected to become more stringent (and difficult to quantify), as we will discuss in Sec. VI.

In either case, the coefficients of the dimension- 6 operators (12) are proportional to

$$
\zeta \equiv \frac{|\psi(0)|^{2}}{m_{R}^{3}},
$$

\footnotetext{
${ }^{2}$ Also this choice maximizes the wave function at the origin.
} 


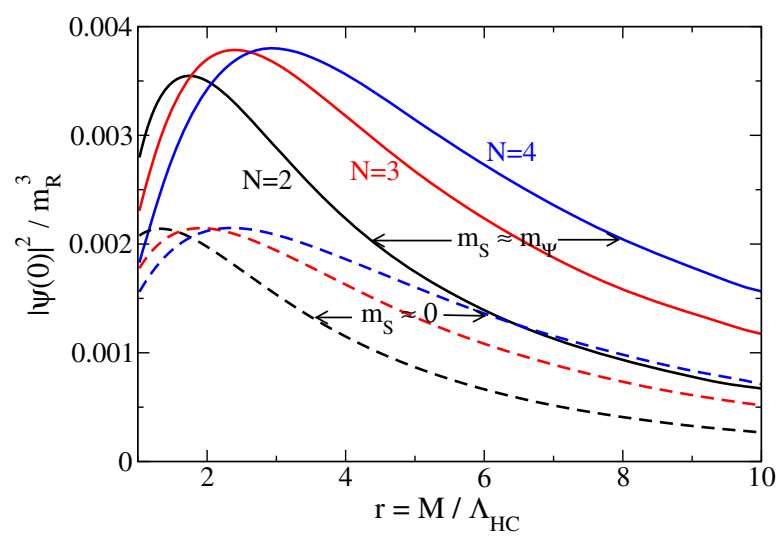

FIG. 2. The function $\zeta=|\psi(0)|^{2} / m_{R}^{3}$ [Eq. (14)] that encodes the strong dynamics dependence of the exchange of bound state resonances between SM fermion bilinears. Solid curves are for all constituents having same mass $M$, while dashed are for the case of $m_{S} \ll \Lambda_{H C}$.

which encodes the effects of the confining dynamics (see Appendix A), where $m_{R}$ is the mass of the exchanged resonance. $\zeta$ is dimensionless and thus only depends upon ratios of mass or energy scales (and $N_{H C}$ ). In the case where all masses are approximately equal to scale $M$, there is just one such ratio, $r=M / \Lambda_{H C}$. Using the nonrelativisitic potential model in the region $M / \Lambda_{H C}>1$, we numerically determine $\zeta(r)$ for $N_{H C}=2,3,4$, shown in Fig. 2 (solid curves). It reaches a maximum value of $\sim 0.0037$ for $r \cong 3$ if $N_{H C}=3,4$, and is smaller if $N_{H C}=2$. For the case $m_{S} \ll \Lambda_{H C}$, the low scale $m_{S}$ becomes irrelevant and we can still express $\zeta$ as a function of the same $r=M / \Lambda_{H C}$, using the relativistic version of the potential model. $\zeta$ is smaller for these models, $\sim 0.002$ for $r \sim(2-3)$.

Since $\zeta$ is small, the best case for avoiding large couplings $\lambda_{2}, \tilde{\lambda}_{2,3}$ in the underlying model is to have $m_{\phi} \sim$ $m_{\Psi} \sim M$ and $\Lambda_{H C} \sim(0.3-0.4) M$ for $N_{H C}=3$ or 4 . Then with $\zeta=0.0037$, Eq. (13) implies

$$
\left|\lambda_{2}^{2} \tilde{\lambda}_{2} \tilde{\lambda}_{3}\right| \cong 0.3\left(\frac{M}{\mathrm{TeV}}\right)^{2}\left(\frac{3}{N_{H C}}\right)
$$

From the potential model, the bound state mass $m_{\rho}$ at $\Lambda / M=$ 0.4 and $N_{H C}=3$ is predicted to be 1.75 times the naive value of $2 M$ that ignores the contribution from the hypercolor flux tube. For $M=1 \mathrm{TeV}$, therefore, $m_{\rho} \cong 3.5 \mathrm{TeV}$.

In the case where $m_{S} \ll \Lambda_{H C}$, the couplings must be increased by $\sim 15 \%$, to compensate for the smaller $\zeta$ factor. From the partially relativistic version of the potential model, the mass of the resonance is found to be approximately

$$
m_{\rho} \cong\left(0.56+1.8 N_{H C}\right) \Lambda_{H C}+m_{\Psi},
$$

valid for $m_{\Psi} / \Lambda_{H C} \gtrsim 3$; for smaller values $m_{\rho}$ tends toward a constant independent of $m_{\Psi}$.

\section{A. Meson-antimeson mixing}

Diagrams of the type Fig. 1(c) contribute to the oscillations of neutral mesons: $K^{0}-\bar{K}^{0}, D^{0}-\bar{D}^{0}, B^{0}-\bar{B}^{0}$ and $B_{s}^{0}-\bar{B}_{s}^{0}$, placing constraints on different combinations of the $\tilde{\lambda}_{i}$ couplings by similar reasoning as led to Eq. (15). For the fiducial model parameters with $\zeta=0.0037$ and $N_{H C}=3$, we find the upper limits given in Table II. These are inferred by comparing the operator coefficients $k_{i j}^{2} / 2 m_{\rho_{\Psi}^{2}}$ with the upper limits from Ref. [16] for $B_{s}$ mixing and Refs. [91-95] for $K, D$, and $B_{d}$ mixing (which gives the most stringent bounds in each case). The bounds on $K$ and $D$ mixing are conservative in the sense that we have assumed that the contribution to the imaginary part of the amplitude, which is much more strongly constrained, is as large as that of the real part.

As an example of parameters that can satisfy these constraints, we take $M=1 \mathrm{TeV}$ and

$$
\begin{array}{rlrl}
\tilde{\lambda}_{1}=-0.01, & \tilde{\lambda}_{2}=0.1, & \tilde{\lambda}_{3}=0.66, & \lambda_{2}=2.1 \\
\left(\tilde{\lambda}_{1}^{\prime}=0.014,\right. & \tilde{\lambda}_{2}^{\prime}=0.13, & \left.\tilde{\lambda}_{3}^{\prime}=0.66\right) .
\end{array}
$$

The primed couplings (pertaining to the up-type quarks) are determined by the unprimed ones through Eq. (5). The resulting contributions to the products of couplings relevant to meson mixing are shown in Table II (last column). The relative minus sign between $\tilde{\lambda}_{1}$ and $\tilde{\lambda}_{2}$ leads to an accidental cancellation in $V_{i j} \tilde{\lambda}_{j}$ such that the $D^{0}$ mixing contribution is just below the limit; without this sign, we would get $\tilde{\lambda}_{1}^{\prime} \tilde{\lambda}_{2}^{\prime}=0.004$, saturating the limit.

The fact that all the mixing constraints are close to being saturated, with the exception of $B_{s}$, can be understood from the lack of any approximate flavor symmetry such as minimal flavor violation (MFV) [96] in this model [although the hierarchical nature of our choice of couplings in (17) functions in a similar way to MFV]. By requiring a large enough $b \rightarrow s \mu^{+} \mu^{-}$transition and trying to avoid couplings much larger than 1 , we are pushed into this restricted region of parameter space.

One way to relieve the tension is to take smaller values of $M$, say $800 \mathrm{GeV}$. Then the couplings could on average be

TABLE II. 3rd column: Bounds from meson-antimeson mixing on quark couplings. $M$ is the (assumed) universal mass of the constituents. Last column: Values following from fiducial parameter choices, Eq. (17).

\begin{tabular}{lccc}
\hline \hline Meson & Quantity & $\begin{array}{c}\text { Upper limit } \\
\text { (units } M / \mathrm{TeV} \text { ) }\end{array}$ & $\begin{array}{c}\text { Fiducial value } \\
\text { (units } M / \mathrm{TeV} \text { ) }\end{array}$ \\
\hline$K^{0}$ & $\left|\tilde{\lambda}_{\hat{\lambda}} \tilde{\lambda}_{2}\right|$ & $1.3 \times 10^{-3}$ & $1 \times 10^{-3}$ \\
$D^{0}$ & $\left|\tilde{\lambda}_{\lambda}^{\prime} \tilde{\lambda}_{2}^{\prime}\right|$ & $2 \times 10^{-3}$ & $7 \times 10^{-4}$ \\
$B^{0}$ & $\left|\tilde{\lambda}_{1} \tilde{\lambda}_{3}\right|$ & 0.026 & 0.0066 \\
$B_{s}^{0}$ & $\left|\tilde{\lambda}_{2} \tilde{\lambda}_{3}\right|$ & 0.066 & 0.066 \\
\hline \hline
\end{tabular}


smaller by a factor of $\sqrt{0.8}$ than in Eq. (17). If we keep the leptonic coupling large, $\lambda_{2}=1.1$, the quark couplings can all be reduced by 0.8 , and the last column in Table II is reduced by 0.64 , leading to a $60 \%$ reduction in the actual amplitude. Allowing for larger leptonic coupling can further ameliorate the situation, which is thus not as marginal as the limiting case makes it appear. $M$ cannot be made lower than $800 \mathrm{GeV}$ because of LHC constraints, as we will see in Sec. VI. Our main goal in this paper is to establish some region of viability for the model. A thorough exploration of the parameter space will become more strongly motivated if the $B$ decay anomalies are confirmed with greater significance.

\section{B. Lepton flavor violating decays}

As mentioned above, nothing obliges us to turn on the couplings $\lambda_{1}, \lambda_{3}$ to $e$ and $\tau$ leptons in this model, apart from aesthetic considerations. If these couplings are nonvanishing, then the diagram of Fig. 1(b) contributes to leptonflavor violating (LFV) decays $\tau \rightarrow 3 \ell$ (where $\ell$ is $\mu$ or $e$ ) and $\mu \rightarrow 3 e$. We can derive upper bounds on $\left|\lambda_{1}\right|,\left|\lambda_{3}\right|$ by comparing the amplitude from exchange of the $\rho_{S}=S \bar{S}$ vector meson,

$$
\frac{3}{4} \zeta \frac{\lambda_{i} \lambda_{j}^{*}\left|\lambda_{j}\right|^{2}}{2 M^{2}}\left(\bar{\ell}_{j} \gamma^{\mu} \ell_{i}\right)\left(\bar{\ell}_{j} \gamma^{\mu} \ell_{j}\right)
$$

to the SM amplitude $2 \sqrt{2} G_{F}\left(\bar{\nu}_{i} \gamma^{\mu} \ell_{i}\right)\left(\bar{\ell}_{j} \gamma^{\mu} \nu_{j}\right)$ for the corresponding allowed decay, giving a ratio of branching ratios of $R_{i j}=1.8 \times 10^{-9}\left|\lambda_{i}\right|^{2}\left|\lambda_{j}\right|^{6}$. From $\tau \rightarrow 3 \mu$ we require $R_{\tau \mu}<1.2 \times 10^{-7}$ and from $\mu \rightarrow 3 e, R_{\mu e}<10^{-12}$, giving

$$
\left|\lambda_{1}\right| \lesssim 0.23, \quad\left|\lambda_{3}\right| \lesssim 0.9 .
$$

This implies that the anomalous contribution to the $b \rightarrow s e^{+} e^{-}$amplitude (which goes as $\left|\lambda_{1}\right|^{2}$ ) is less than 0.01 times that of $b \rightarrow s \mu^{+} \mu^{-}$.

\section{Radiative transitions}

Another potentially important class of FCNCs is the radiative decays such as $b \rightarrow s \gamma, \tau \rightarrow \mu \gamma$. They arise through the mass mixing of SM fermions with heavy composite fermions from the confining sector. These are vectorlike doublet leptons and quarks, $\phi S \equiv F_{\ell}$ and $\phi \Psi \equiv F_{q}$, that naturally have magnetic moments coming from their charged constituents. They contribute to dipole operators of the light fermions in the mass eigenbasis, including transition magnetic moments.

The mass mixing in this kind of model was previously considered in Ref. [97]. Here we make the connection to transition moments more precise, using methods that were previously applied to the rare decays of $Z \rightarrow \Upsilon_{\gamma}$ in the SM in Ref. [98]. The contributions look like loop diagrams

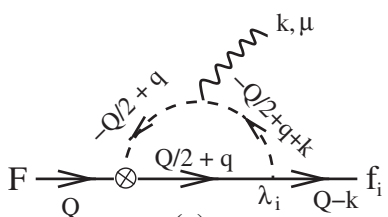

(a)

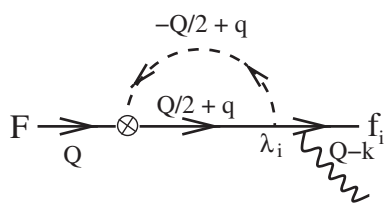

(c)

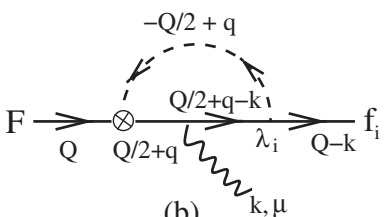

(b)

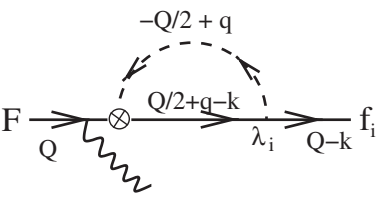

(d)
FIG. 3. Diagrams for transition magnetic moment from heavy composite to light fundamental fermion. Equal constituent masses are assumed for routing of momenta. Cross represents the bound-state wave function.

(Fig. 3), but the integral is over the relative momentum of the bound constituents and is weighted by the wave function in momentum space. Details are given in Appendix B. There we show that the mass-mixing between the SM and heavy fermions takes the form

$$
\begin{aligned}
& \tilde{\lambda}_{f} \bar{Q}_{f, a} \phi^{a} \Psi+\lambda_{i} \bar{S} \phi_{a}^{*} L_{i}^{a} \rightarrow \frac{\psi(0)}{\sqrt{M}}\left(\tilde{\lambda}_{i} \bar{Q}_{i} F_{q}+\lambda_{i} \bar{F}_{\ell} L_{i}\right) \\
& \equiv \mu_{q}^{i} \bar{Q}_{i} F_{q}+\mu_{\ell}^{i} \bar{F}_{\ell} L_{i},
\end{aligned}
$$

where $\psi$ is the wave function of the bound state and $M$ is the mass of the constituents, which we continue to take to be equal for simplicity. For unequal masses, $M$ is replaced by twice the reduced mass.

In addition, as shown in Appendix B, the diagrams of Fig. 3 give rise to dimension- 6 transition moment operators of the form

$$
\mathcal{L}=\frac{-e F^{\mu \nu}}{2 M_{F}^{2}}\left(q_{q} \mu_{q}^{f} \bar{Q}_{f} \sigma_{\mu \nu} F_{q}+q_{\ell} \mu_{\ell}^{f} \bar{F}_{\ell} \sigma_{\mu \nu} L_{f}\right),
$$

where $q_{f}$ is the electric charge of particle $f$, a quark or lepton. Reference [99] noted that these lead to transition magnetic moments among the light fermion states upon diagonalization of the mass matrices, which take the form

$$
\left(\begin{array}{ll}
\bar{f}_{R} & \bar{F}_{R}
\end{array}\right)\left(\begin{array}{cc}
m_{f} & 0 \\
\mu_{f} & M_{F}
\end{array}\right)\left(\begin{array}{l}
f_{L} \\
F_{L}
\end{array}\right),
$$

where $f, F$ denote the SM and heavy fermions, respectively, with $m_{f}$ being the SM mass matrix and $M_{F}$ the heavy composite mass. To leading order for small mixing, (22) is diagonalized by separate left- and right-handed transformations $\mathcal{O}_{L, R}=\left(\begin{array}{cc}1 & \theta_{L, R} \\ -\theta_{L, R}^{T} & 1\end{array}\right)$ with mixing angles that are vectors in flavor space,

$$
\theta_{R}=\frac{m_{f} \mu_{f}}{M_{F}^{2}}, \quad \theta_{L}=\frac{\mu_{f}}{M_{F}} .
$$


After transforming to the mass eigenbasis, a transition moment for the light fermions is generated,

$$
\mathcal{L}=e \frac{q_{f} \mu_{f}^{i} \mu_{f}^{j} m_{f}^{j}}{2 M_{F}^{4}}\left(\bar{f}_{L, i} \sigma_{\mu \nu} f_{R, j}\right) F^{\mu \nu}
$$

It is proportional to $|\psi(0)|^{2} / M_{F}^{4}=\zeta / M_{F}$ rather than just $\zeta$ [recall Eq. (14)]. The same potential model can be used to compute $M_{F}$ as we used for the bosonic bound states, since quantum mechanics is insensitive to the spins of the constituent particles, and we are ignoring the spin-spin interactions in our simple treatment. Then for the fiducial parameters with $\Lambda_{H C}=0.4 M,|\psi(0)|^{2} / M_{F}^{4} \cong \zeta /(3.5 M)$.

\section{FCNC quark transitions}

From the general formula (24), the operator contributing to $b \rightarrow s \gamma$ is

$$
\frac{e \tilde{\lambda}_{2} \tilde{\lambda}_{3}|\psi(0)|^{2} m_{b}}{6 M_{F_{d}}^{4} M} \bar{s}_{L} q \gamma^{\mu} b_{R}
$$

This implies that the conventionally normalized Wilson coefficient of the dipole operator $\mathcal{O}_{7}$ is

$$
C_{7}=\frac{\sqrt{2} \pi^{2} \tilde{\lambda}_{2} \tilde{\lambda}_{3} \zeta}{10.5 G_{F} V_{t s} V_{t b} M^{2}} \cong 7 \times 10^{-4},
$$

using the parameter values (17) with $\Lambda_{H C}=400 \mathrm{GeV}$, well below the experimental limit of $\sim 0.02$ [100]. Under the scaling of parameters described at the end of Sec. IVA, this prediction remains unchanged.

Similarly for $c \rightarrow u \gamma$, we predict a transition magnetic moment like (25) with $\tilde{\lambda}_{i} \rightarrow \tilde{\lambda}_{i}^{\prime}$, Eq. (5). Using the values (17), the contribution to the $c \rightarrow u \gamma$ amplitude is $\sim 10^{-2}$ smaller than the SM contribution. Likewise, the contribution to $d \rightarrow s \gamma$ is orders of magnitude below the limit from $K_{S} \rightarrow \pi^{0} \ell^{+} \ell^{-}$decays (see Sec. VII.7 of [101]).

\section{FCNC leptonic decays}

The radiative decays $\ell_{i} \rightarrow \ell_{j} \gamma$ proceed analogously to those of the quarks, due to mixing with the heavy composite lepton whose mass is $M_{F_{\ell}} \cong 3.5 \mathrm{M}$ for our fiducial parameter choice. The induced transition magnetic moment is

$$
\mu_{i j}=\frac{e \lambda_{i} \lambda_{j} \zeta m_{i}}{M_{F_{\ell}} M} .
$$

and the partial decay width is $\delta \Gamma=\mu_{i j}^{2} m_{i}^{3} / 8 \pi$ [102]. This gives limits

$$
\left|\lambda_{1}\right| \lesssim 7.5 \times 10^{-4}, \quad\left|\lambda_{3}\right| \lesssim 0.56,
$$

respectively, from $\mu \rightarrow e \gamma$ and $\tau \rightarrow \mu \gamma$ that are more stringent than (19).

\section{Muon anomalous magnetic moment}

The contribution to the muon anomalous magnetic moment is

$$
a_{\mu}=\frac{(g-2)}{2}=\left|\lambda_{2}\right|^{2} \frac{m_{\mu}^{2} \zeta}{M_{F_{\ell}} M} .
$$

For our benchmark values, we get $a_{\mu} \sim 10^{-11}$, which is 300 times too small for addressing the current discrepancy with predictions of the SM [103]. It will be seen below that LHC constraints prevent taking $M_{F_{\ell}}$ to be smaller than the $\mathrm{TeV}$ scale, leaving little room for increasing $a_{\mu}$.

\section{Precision electroweak constraints}

The composite vector states $\rho_{\phi}^{\mu}$ made from $\phi^{a} \phi_{b}^{*}$ can be a singlet or a triplet of $\mathrm{SU}(2)_{L}$. The latter have mass mixing with the SM electroweak gauge bosons, induced by diagrams analogous to those in Fig. 3, without the external photon line, with $\rho_{\phi}^{\mu}$ and $W^{\mu}$ as the external states, and $\phi / \phi^{*}$ as the internal ones. The amplitude for $\rho_{\phi} \rightarrow W$, which is related to the off-diagonal element $m_{\rho_{\phi} W}^{2}$ in the mass matrix, is

$$
\mathcal{A}\left(\rho_{\phi} \rightarrow W\right)=\frac{2 g_{2}}{\sqrt{m_{\phi}}} \vec{\epsilon} \cdot \nabla \psi(0),
$$

where $g_{2}$ is the weak gauge coupling, $\vec{\epsilon}$ is the $W$ polarization vector, and $|\nabla \psi(0)|^{2} / m_{\rho_{\phi}}^{5}=\left(\mu_{*} / m_{\rho_{\phi}}\right)^{5} / 96 \pi \cong$ $5 \times 10^{-5}$ from the potential model for $p$-wave states, evaluated at $\Lambda_{H C}=0.4 m_{\phi}$. For our fiducial parameter choices, this leads to $m_{\rho_{\phi} W}^{2} \cong 0.34 \mathrm{TeV}^{2}$, while the diagonal element is $m_{\rho_{\phi}}^{2} \cong 19.0 \mathrm{TeV}^{2}$.

Naively this would lead to a large shift in $m_{W}^{2}$, but as noted in Ref. [88], models like ours respect custodial symmetry, so that the electroweak precision PeskinTakeuchi parameters [104] $T$ and $U$ vanish. Moreover, $S=0$ so long as the new physics does not contribute to electroweak symmetry breaking, since in this case it induces no kinetic mixing of the form $W_{3}^{\mu \nu} Y_{\mu \nu}$ between the neutral SM gauge bosons. In our model this could come only from the composite scalar triplet that is also a bound state of $\phi \phi^{*}$. However, its squared mass is positive, coming largely from the constituent masses, so it does not get a vacuum expectation value. Reference [78] explicitly confirmed these statements in a related model having composite vector bosons that mix with $W$ and $Z$.

\section{E. Higgs coupling strengths}

Because the SM fermions mix with the heavy fermionic partners, the light fermion mass eigenstates are admixtures of the two fields, whereas only the SM component couples to the Higgs boson. The coupling of the Higgs to fermions is thus reduced by mixing angle factors 

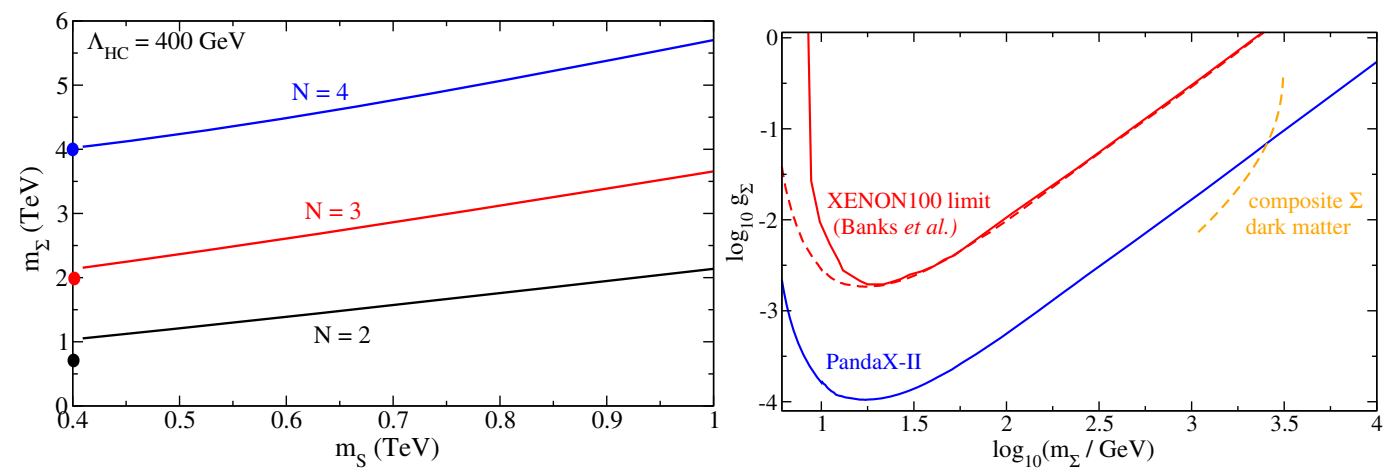

FIG. 4. Left: Hyperbaryonic dark matter mass $m_{\Sigma}$ versus constituent mass $m_{S}$ for $N_{H C}=2,3,4$ and $\Lambda_{H C}=400 \mathrm{GeV}$. Heavy dots show the value of $m_{\Sigma}$ in the limit $m_{S} \rightarrow 0$. Right: Predicted gyromagnetic moment for the $N_{H C}=3$ model (orange dashed) and current constraint from direct detection [106] (solid blue), based on rescaling of earlier limit from Ref. [107] (solid red). Dashed red is an estimate of the improved relative sensitivity at low mass in recent experiments, compared to the XENON 100 [108] limit.

$$
\bar{f}_{L} h f_{R} \rightarrow \cos \theta_{L} \cos \theta_{R} f_{L}^{\prime} h f_{R}^{\prime} .
$$

Here the primed fields are the mass eigenstates, and $\theta_{L, R}$ are the mixing angles given in Eq. (23). The largest deviations come from the $\theta_{L}$ for the fermions with the largest $\lambda_{i}$ or $\tilde{\lambda}_{i}$ couplings, namely $\mu, b, t$ and possibly $\tau$. Denoting the coupling generically by $\lambda$, we find $\theta_{L} \cong 0.1 \lambda$, leading to a reduction in the Higgs coupling strength by a factor of $\kappa=1-0.005 \lambda^{2}$. This is too small a deviation compared to the current sensitivity of LHC experiments to be constraining.

\section{COMPOSITE DARK MATTER}

The $\eta^{\prime}$-like $S \bar{S}$ bound state is unstable to self-annihilation into $\mu^{+} \mu^{-}$through $\phi$ exchange, but the hyperbaryonic bound state $\Sigma \equiv S^{N}$ is a stable dark matter candidate. Determination of its thermal relic density can depend upon the annihilation of the constituent $S \bar{S}$ particles above the $\mathrm{SU}\left(N_{H C}\right)$ confinement transition, as well as $\Sigma \bar{\Sigma}$ annihilations following confinement. For the parameters of interest, with $\Lambda_{H C} \sim 400 \mathrm{GeV}$ and $m_{S} \lesssim 1 \mathrm{TeV}$, it has been shown $[97,105]$ that the resulting relic density is too small (by a factor of 1000). However, since hyperbaryon number is conserved, ${ }^{3}$ it is possible that an asymmetry between $S$ and $\bar{S}$ was produced in the early universe, so $\Sigma$ can be asymmetric dark matter. We make this assumption here, without attempting to account for the origin of the asymmetry.

From the nonrelativistic potential model, the DM mass can be numerically determined as a function of $m_{S}$. In the range $1<m_{S} / \Lambda_{H C}<10$, we find the approximate fit

$$
m_{\Sigma} \cong\left(3.8\left(1-N_{H C}\right)+N_{H C}^{2}\right) \Lambda_{H C}+\left(0.3+0.8 N_{H C}\right) m_{S}
$$

\footnotetext{
${ }^{3}$ Note that $S, \Psi$, and $\phi^{*}$ can be assigned hyperbaryon number +1 .
}

The dependence on $m_{S}$ is shown in Fig. 4 (left), including the limit $m_{S} \rightarrow 0$ (heavy dots on $y$ axis), obtained from the relativistic version of the potential model.

Although $S$ has no direct interactions with nuclei, it gets a magnetic moment at one loop (with $\phi^{ \pm}$or $\mu^{ \pm}$in the loop), depending upon $R \equiv m_{S}^{2} / m_{\phi}^{2}$,

$$
\mu_{S}=\frac{e\left|\lambda_{2}\right|^{2} m_{S}}{32 \pi^{2} m_{\phi}^{2}} f(R),
$$

with

$$
f(R)=\int_{0}^{1} d u\left(\frac{u^{2}}{1-R u}+\frac{u(1-u)}{1-R+R u}\right)
$$

in the approximation of neglecting $m_{\mu}$ (see Appendix C for details). The loop function diverges logarithmically as $R \rightarrow 1$ in this approximation; for example, if $m_{S}=0.9 m_{\phi}$, $f(0.81)=1.3$, while for $m_{S}=0.8 m_{\phi}, f(0.64)=0.93$.

If $\Sigma$ has spin, it inherits a magnetic moment from its $S$ constituents. This will be the case if $N_{H C}$ is odd. If $N_{H C}=3$, the wave function of $S^{a} S^{b} S^{c}$ is $\epsilon_{a b c}$ in hypercolor space, and if the spatial wave function is $s$-wave, total antisymmetry demands that the spins be aligned, like the $\Delta$ baryon in QCD. The quark model then predicts that $\Sigma$ has a magnetic moment that is three times that of $S$.

The gyromagnetic ratio of $\Sigma$ is given (for odd $N_{H C}$ ) by $g_{\Sigma}=4 N_{H C} \mu_{s} M_{\Sigma} / e$. Reference [107] derived an upper limit on $g$ versus DM mass based upon early XENON100 data [108]. We update their limit by comparing the relative sensitivities of that search to the recent PandaX-II result [106] in the high mass regime, to constrain our model. Allowing $m_{S}$ to vary between $\Lambda_{H C} \cong 400 \mathrm{GeV}$ (the value that maximizes $\zeta$ if $M=1 \mathrm{TeV}$ ) and $1 \mathrm{TeV}$, we rescale $\lambda_{2}^{2}$ by $\left(\zeta_{\max } / \zeta\right)^{1 / 2}$ where $\zeta_{\max }=0.0037$ for the fiducial model parameters, and $\zeta$ is smaller for $m_{S}<M$. This keeps the predicted $B$ decay signals constant while varying $m_{S}$, if all the couplings scale in the same way. Using (32), $g_{\Sigma}$ and $m_{\Sigma}$ 
are determined as a function of $m_{S}$. The result for $N_{H C}=3$ is shown in Fig. 4 (right). It translates to an upper limit $m_{S} \lesssim 800 \mathrm{GeV}$. ( $\zeta$ is not appreciably decreased at this value of $m_{S}$.)

\section{COLLIDER CONSTRAINTS}

Because of hypercolor confinement, only HC-neutral bound states of the new particles $\phi, \Psi, S$ can be produced in high-energy collisions. We expect the dominant mode for discovery to be resonant production of vector and scalar $\mathrm{HC}$ mesons, through the diagrams in Fig. 5. We work in a region of parameter space where $\Lambda_{H C}$ is not too much lower than the constituent masses $M$, which avoids the complications of quirks [89]. In the quirky regime, there are many excited quantum states to scatter into, that can efficiently radiate hypergluons and settle to the ground state before self-annihilating. Then partons with energy above that of the resonance could still be effective for production.

On the other hand for $\Lambda_{H C} \lesssim M$, the resonances are wellseparated and can only radiate QCD gluons or electroweak gauge bosons since the $\mathrm{HC}$ glueball mass is too heavy, $\sim 7 \Lambda_{H C}$ [109] for $N_{H C}=3$. In this regime, the parton center of mass energy must match the mass of the resonance, and we can use similar techniques for computing resonant production of bound states as for $J / \psi$ and $\Upsilon$ in $e^{+} e^{-}$ collisions. This approximation misses events in which the bound state is produced by partons above the threshold for the resonance, plus QCD radiation to take away the excess energy. We defer study of such higher-order corrections.

\section{A. Production cross section}

In general, the production cross section from colliding protons to form a narrow resonance $R$ from partons $p_{i}, p_{j}$ can be expressed as

$$
\begin{aligned}
\sigma(p p \rightarrow R)= & \frac{16 \pi^{2}}{m_{R} s} \frac{(2 J+1) N_{c} \Gamma\left(R \rightarrow p_{i} p_{j}\right)}{\left(2 s_{1}+1\right)\left(2 s_{2}+1\right) N_{c 1} N_{c 2}} \\
& \cdot \int_{m_{R} / s}^{1} \frac{d x}{x}\left[f_{i}(x) f_{j}\left(m_{R} / s x\right)+\{i \leftrightarrow j\}\right],
\end{aligned}
$$

where $f_{i}$ is the parton distribution (PDF) for $p_{i}, 2 s_{i}+1$ and $N_{c i}$ are the number of spin states and colors, respectively, of the incoming particles, $2 J+1$ and $N_{c}$ likewise for the

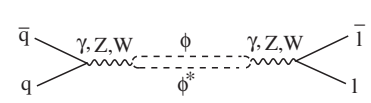

(a)

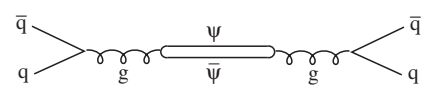

(c)

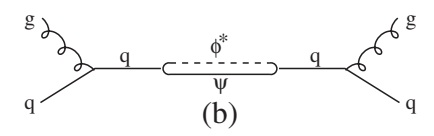

(b)

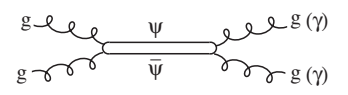

(d)
FIG. 5. Resonant production of HC bound states leading to dileptons (a), dijets (b)-(d), or diphotons (d). resonance, and $\Gamma$ is the partial width for the decay that is inverse to the production process. (In the case of incoming gluons, the doubling of PDFs in the second line is correct, compensating for the factor of $1 / 2$ in the phase space for decay of $R$ into identical particles.) The generalization to resonances carrying $\mathrm{SU}(2)_{L}$ rather than $\mathrm{QCD}$ quantum numbers is obvious.

\section{B. Predicted widths}

The decay widths of bound states have been quantitatively addressed in regimes where the constituent masses are either much heavier than the confinement scale [89] or much lighter $[88,110]$. We are interested in the case where $\Lambda_{H C} \sim 0.4 M$ (with the possible exception of $m_{S} \ll \Lambda_{H C}$, but this is not relevant here because the $S \bar{S}$ bound states cannot be produced at LHC). For $N_{H C}=3$, the potential model predicts that the kinetic energy of the constituent is 0.3 of its mass energy, so the nonrelativistic approximation is not very bad. Ultimately, lattice calculations should be done to make more quantitative predictions.

We ignore decays of bound states into hypergluons, since we work in a regime where any hyperhadrons are too heavy to be produced. Even if $m_{S}<\Lambda_{H C}$, the $\Pi_{S}=S \bar{S}$ pseudoscalar is not a light pseudo-Nambu-Goldstone boson, because the approximate chiral U(1) flavor symmetry is anomalous, like for the $\eta^{\prime}$ of QCD. Likewise, the glueballs of the $\mathrm{SU}\left(N_{H C}\right)$ are also too heavy.

Reference [89] calculates (in terms of $|\psi(0)|^{2}$ ) the decay widths assuming that the bound state constituents do not form a resonance of definite spin, but these are straightforward to rescale for the physical eigenstates of spin. ${ }^{4}$ Doing this gives results in agreement with the treatment of bound state decays in Ref. [111]. In our model, the $\Psi \bar{\Psi}$ states can be in the QCD color singlet or octet representations. Given that $\Lambda_{H C} \gg \Lambda_{\mathrm{QCD}}$, this distinction is unimportant for the dynamics since the lifetime of the bound state is much shorter than the QCD hadronization time scale.

\section{1. $\Psi \bar{\Psi}$ resonances}

Starting with the $\Psi \bar{\Psi}$ bound states, there is the pseudoscalar $\Pi_{\Psi}$ that can be produced by gluon fusion [diagram (d) of Fig. 5] or the vector $\rho_{\Psi}$ coming from $q \bar{q}$ annihilation, Fig. 5(c). The respective decay widths are [89]

$$
\Gamma\left(\Pi_{\Psi} \rightarrow g g\right)=\frac{128 \pi}{27} N_{H C} \alpha_{s}^{2} \frac{|\psi(0)|^{2}}{m_{\Pi_{\Psi}}^{2}}
$$

\footnotetext{
${ }^{4}$ For example, decay via a single virtual gauge boson into fermions $f \bar{f}$ gets a factor of $4 / 3$ to correct for the fact that only the spin- 1 state contributes in the average over spins of the initial constituents. Similarly, decays to two gauge bosons occur only for spin-0 resonances, requiring a factor of 4 correction to the spin-averaged rate. Likewise, the results of [89] ignore QCD color correlations of the constituents and must be rescaled for states of definite color.
} 


$$
\begin{gathered}
\Gamma\left(\rho_{\Psi} \rightarrow u \bar{u}\right)=\frac{8 \pi}{27} N_{H C} \alpha_{s}^{2} \frac{|\psi(0)|^{2}}{m_{\rho_{\Psi}}^{2}} \\
\Gamma\left(\rho_{\Psi} \rightarrow e^{+} e^{-}\right)=\frac{4 \pi}{9} N_{H C} \alpha^{2} \frac{|\psi(0)|^{2}}{m_{\rho_{\Psi}}^{2}} .
\end{gathered}
$$

The last one, unlike the previous two, involves only the color singlet state so it does not entail an average over QCD colors. We include it because it is potentially relevant for the dilepton final state. However, counting channels, it predicts a branching ratio of $B_{l^{+} l^{-}}=\left(\alpha / \alpha_{s}\right)^{2} / 2 \cong$ $2.5 \times 10^{-7}$ into electrons or muons, implying that the dilepton channel is actually unimportant for this resonance.

\section{2. $\phi \bar{\phi}$ resonance}

There are four kinds of $\rho_{\phi}=\phi \phi^{*}$ vector bound states, three components in an $\mathrm{SU}(2)_{L}$ triplet and one singlet. Since the $\mathrm{SU}(2)_{L}$ dynamics are not important at the $\mathrm{TeV}$ scale, we average over the isospins of the bound state constituents. The angular momentum of $\rho_{\phi}$ is purely orbital, so the constituents are in a relative $p$-wave for which $\psi(0)=0$. Therefore, the width depends upon the derivative of $\psi$ at the origin. Following [112], we find that the $\mathrm{SU}(2)_{L}$ contribution to the width is

$$
\Gamma\left(\rho_{\phi} \rightarrow W^{*} \rightarrow q_{i} \bar{q}_{j}\right)=\frac{3 \pi}{2} N_{H C} \alpha_{2}^{2} \frac{|\vec{\nabla} \psi(0)|^{2}}{m_{\rho_{\phi}}^{4}}
$$

for decay into a single generation of approximately massless quarks. For decays into leptons, the result is $1 / 3$ times smaller (from lack of QCD color), giving a branching ratio of $3 / 4$ to quarks and $1 / 4$ to leptons. Hence, this process leads to both dijet and dilepton signals. For decays into muons, there is an additional, potentially larger contribution from $S$ exchange (that interferes with the $W$ exchange contribution, but we ignore this interference for purposes of estimation), given by

$\Gamma\left(\rho_{\phi} \rightarrow \mu^{+} \mu^{-}\right)=\frac{2 \pi}{3} N_{H C}\left(\frac{\left|\lambda_{2}\right|^{2}}{4 \pi}\right)^{2} \frac{|\vec{\nabla} \psi(0)|^{2}}{\left(\frac{1}{4} m_{\rho_{\phi}}^{2}+m_{S}^{2}\right)^{2}}$.

\section{Composite fermion}

For decay of the composite fermion to $q g$, taking $m_{\phi} \cong m_{\Psi}$ as before, we find

$$
\Gamma\left(F_{q} \rightarrow g q_{i}\right) \cong N_{H C} \frac{\alpha_{s}\left|\tilde{\lambda}_{i}^{(\prime)}\right|^{2}}{2 m_{\Psi}^{2}}|\psi(0)|^{2}
$$

where $i$ is the generation index of the quark and, as usual, the prime is for couplings to up-type quarks. The squared coupling must be absorbed into sum over quark flavors in the parton luminosity factor for production via $q g$ fusion.

\section{Vertex correction}

For all of the annihilation decays, a more quantitative estimate can be made by taking account of perturbative corrections that dress the annihilation vertex. For charmonium and upsilon, the correction from a gluon loop is known to be important, and we adopt the analogous correction for the HC gluon exchange, with $\alpha_{H C}$ evaluated at the scale of the constituent mass. The correction factors depend upon the annihilation process, and in analogy with QCD we take [113]

$C=1-\frac{\left(N^{2}-1\right) \alpha_{H C}}{8 \pi}\left\{\begin{array}{cc}\left(20-\pi^{2}\right) / 3, & \Pi_{\psi} \rightarrow g g \\ 16 / 3, & \rho_{\psi} \rightarrow f \bar{f}\end{array}\right\}$,

with $\alpha_{H C}$ evaluated at the scale $\mu_{*}$, twice the inverse Bohr radius. We have assumed that the known $N_{H C}=3$ results generalize to other values by rescaling by the number of hypergluons.

\section{Resonant search constraints}

Combining the decay widths with the general formula for the production cross section (35), we find that it depends upon the same combination $\zeta$, Eq. (14), that appeared in the dimension-6 FCNC operators. One finds a production cross section for the color octet plus singlet states $\Pi_{\Psi}$ given by

$$
\sigma\left(p p \rightarrow \Pi_{\Psi}\right)=\frac{8 \pi^{3} \alpha_{s}^{2} N_{H C}}{3 s} \zeta C \mathcal{L}_{g g}
$$

where $\mathcal{L}_{g g}$ is the parton luminosity factor for gluon fusion. The perturbative correction (42) is $C=0.5$ at $m_{\Psi}=2.5 \Lambda_{H C}$, similar to the value that occurs in QCD for $J / \psi$, and $\zeta=0.0037$ as in the previous sections. In Fig. 6 we plot this prediction along with current constraints from ATLAS [114] and CMS [115] resonant dijet searches, assuming an acceptance of events passing experimental cuts of $A=50 \%$, comparable to that of various models tested in the searches. The resonance mass must exceed $2.3 \mathrm{TeV}$, which is satisfied for our fiducial model with $m_{\Pi}=3.6 \mathrm{TeV}$. Keeping $m_{\Psi} / \Lambda_{H C}$ fixed, this limit would allow $m_{\Psi}$ to be no lower than $650 \mathrm{GeV}$.

Similarly, for production of the color octet vector $\rho_{\Psi}$ resonance for $N_{H C}=3$ we find

$$
\sigma\left(p p \rightarrow \rho_{\Psi}\right)=\frac{64 \pi^{3} \alpha_{s}^{2} N_{H C}}{9 s} \zeta C \mathcal{L}_{q \bar{q}} .
$$

The loop correction factor (42) is 0.7 for this process. The corresponding prediction is also plotted in Fig. 6. We infer a limit of $m_{\Psi}>820 \mathrm{GeV}$ for the fiducial model. At larger values of $m_{\Psi} / \Lambda_{H C}=5,10$, this limit becomes more stringent, $m_{\Psi} \gtrsim 1 \mathrm{TeV}$, approximately independent of the value of $m_{\Psi} / \Lambda_{H C}$. 


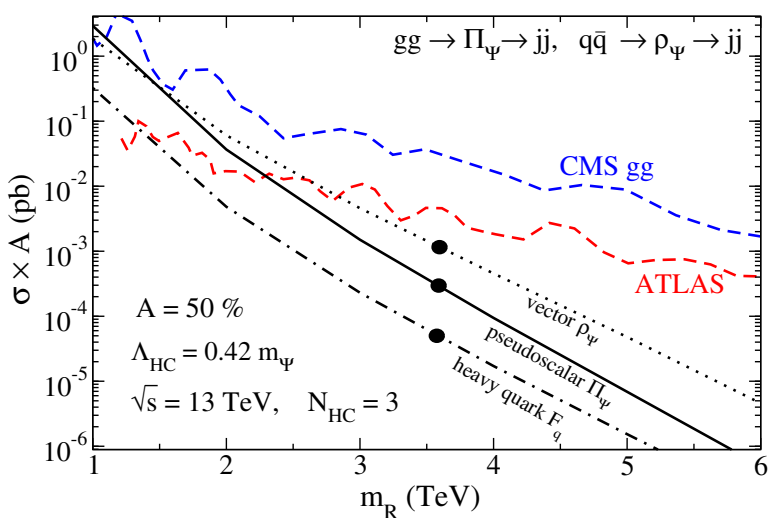

FIG. 6. Dashed curves: Limits on resonant dijet production cross section times acceptance versus resonance mass from ATLAS [114] and CMS [115], along with predictions for $\Pi_{\Psi}$ pseudoscalar (solid curve), $\rho_{\psi}$ vector (dotted curve), and heavy quark $F_{q}$ (dotted-dashed curve) bound states, in the case of $N_{H C}=3$, acceptance $A=100 \%$, and $\sqrt{s}=13 \mathrm{TeV}$ center of mass energy. Predictions for the optimal value (in terms of LHC sensitivity) $\Lambda_{H C} / m_{\Psi}=0.4$ are shown.

In addition to the $\Pi_{\Psi} \rightarrow g g$ channel, the color singlet pseudoscalar can decay to two photons. Its decay width into gluons is smaller than the color average (36) by a factor of $16 / 9$, and its branching ratio into photons is $B_{\gamma \gamma}=$ $9 \alpha^{2} q_{\psi}^{4} / 2 \alpha_{s}^{2}$ [116]. The cross section for $p p \rightarrow \Pi_{\Psi} \rightarrow \gamma \gamma$ is therefore $(9 / 128) B_{\gamma \gamma}$ times Eq. (43). The predicted cross section is shown along with the ATLAS diphoton limit [117] in Fig. 7; it constrains $m_{\Pi}>1.7 \mathrm{TeV}$.

The $\rho_{\phi}$ resonance has the electroweak production cross section

$$
\sigma\left(p p \rightarrow \rho_{\phi}\right)=\frac{2 \pi^{3} \alpha_{2}^{2} N_{H C}}{s} \zeta_{p} \mathcal{L}_{q \bar{q}},
$$

where $\zeta_{p}=|\nabla \psi(0)|^{2} / m_{\rho_{\phi}}^{5}=\left(\mu_{*} / m_{\rho_{\phi}}\right)^{5} / 96 \pi \cong 5 \times 10^{-5}$ from the potential model for $p$-wave states, evaluated at

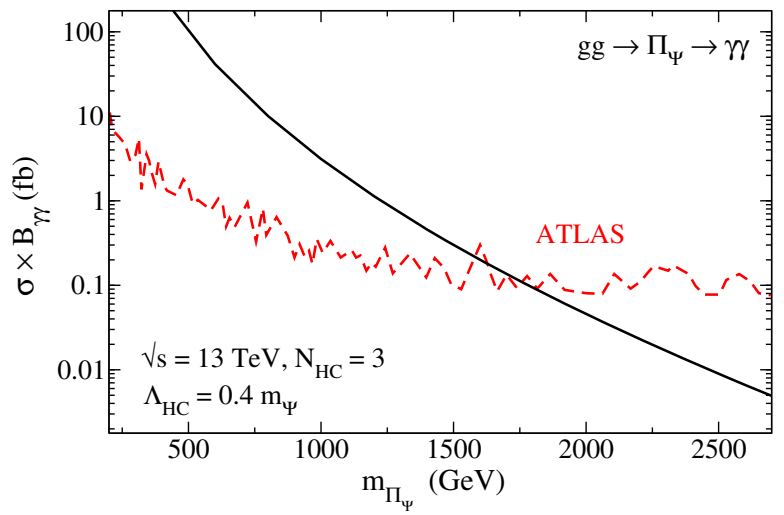

FIG. 7. Production cross section for pseudoscalar bound state $\Pi_{\Psi}$ times branching ratio for decay to two photons. Dashed curve is ATLAS limit [117], solid curve is predicted value for $N_{H C}=3$ and $\Lambda_{H C} / m_{\Psi}=0.4$.

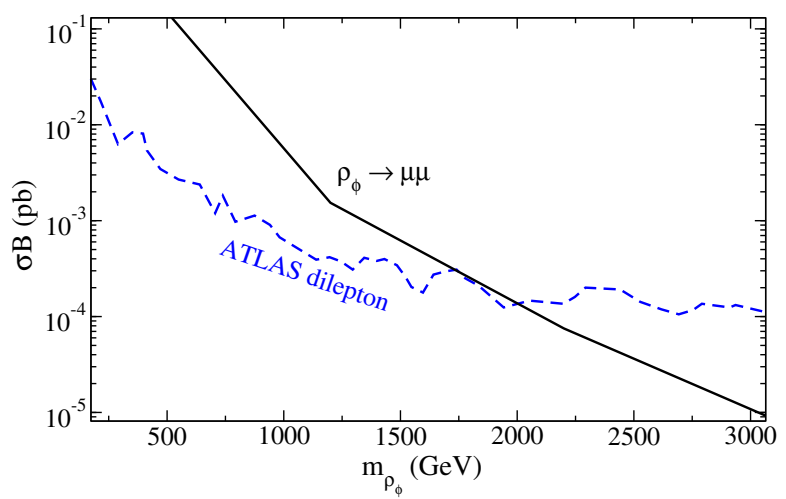

FIG. 8. Production cross section for pseudoscalar bound state $\rho_{\phi}$ times branching ratio for decay to two leptons. Dashed curve is ATLAS limit [118], solid curve is predicted value for $N_{H C}=3$ and $\Lambda_{H C} / m_{\Psi}=0.4$.

$\Lambda_{H C}=0.4 m_{\phi}$. The ATLAS dilepton limit [118], slightly rescaled to account for dominant decay of $\rho_{\phi}$ to muons (see Ref. [49] for details) is plotted in Fig. 8 along with the prediction for a branching ratio of $100 \%$ into $\mu^{+} \mu^{-}$ (this neglects decays into electroweak gauge bosons). The resulting limit $m_{\rho_{\phi}}>2 \mathrm{TeV}$ is stronger than that from diphotons but weaker than that from dijets for the $\Pi_{\Psi}$ and $\rho_{\Psi}$ states, respectively.

The cross section for heavy quark $F_{q}$ production is

$$
\sigma\left(p p \rightarrow F_{q}\right)=\frac{N_{H C} \alpha_{s}}{2 s} \zeta \sum_{i}\left|\tilde{\lambda}_{i}^{(\prime)}\right|^{2} \mathcal{L}_{g q_{i}} .
$$

The result assuming couplings (17) and $100 \%$ branching into dijets is plotted in Fig. 6, giving a weaker constraint than those from $\rho_{\Psi}$ and $\Pi_{\Psi}$ resonant production.

\section{Pair production of bound states}

The wave function at the origin (the $\zeta$ factor) suppresses the predicted resonant production significantly below the cross section that would govern the creation of free pairs of hypercolored constituents. This penalty is avoided if bound states are pair-produced by hadronization in the HC sector, as depicted in Fig. 9. Then each possible kind of final state will occur with a relative probability not much smaller than
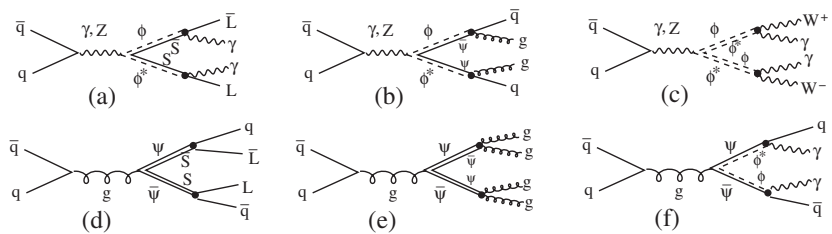

(e)

(f)
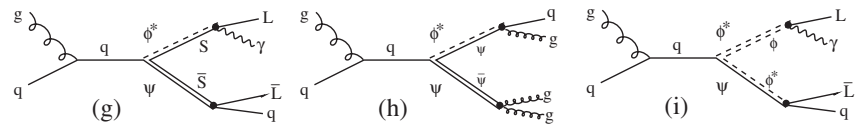

FIG. 9. Pair production of composite states. 
1 since for every pair there are only three kinds of particles $(\Psi, \phi, S)$ that can appear from the vacuum. The only other bottleneck is that the incoming partons must have at least enough energy to make both bound states. Then the cross section averaged over PDFs takes the form

$\sigma=\int_{0}^{1} d x_{1} \int_{0}^{1} d x_{2} f_{1}\left(x_{1}\right) f_{2}\left(x_{2}\right) \Theta\left(x_{1} x_{2} s-4 m_{c}^{2}\right) \hat{\sigma}$,

where $m_{c}$ is the mass of the composite state. The largest cross sections are for $q \bar{q} \rightarrow \Psi \bar{\Psi}$ and $g g \rightarrow \Psi \bar{\Psi}$,

$$
\begin{gathered}
\hat{\sigma}_{q \bar{q}}=\frac{8 \pi \alpha_{s}^{2} N_{H C}}{27 \hat{s}^{2}}\left(2 m_{\Psi}^{2}+\hat{s}\right) \chi, \\
\hat{\sigma}_{g g}=\frac{\pi \alpha_{s}^{2} N_{H C}}{3 \hat{s}}\left(-\frac{\chi}{4}\left(7+31 m_{\Psi}^{2} / s\right)\right. \\
\left.+\left(1+4 m_{\Psi}^{2} / \hat{s}+m_{\Psi}^{4} / \hat{s}^{2}\right) \ln \left(\frac{1+\chi}{1-\chi}\right)\right),
\end{gathered}
$$

with $\chi=\sqrt{1-4 m_{\Psi}^{2} / \hat{s}}$ and $\hat{s}=x_{1} x_{2} s$.

We find that the composite mass $m_{c}$ must be as small as possible to get interesting constraints from this process. This occurs for $m_{S} \ll \Lambda_{H C}$ in the $\Psi \bar{S}$ bound states, for which the potential model gives

$$
m_{c} \cong\left(0.75+1.8 N_{H C}\right) \Lambda_{H C}+m_{\Psi}
$$

These are leptoquark states that decay into $q \ell$, and have been searched for by ATLAS [119] and CMS [120,121]. The searches look for two jets and two leptons, either $\mu^{+} \mu^{-}$or $\tau^{+} \tau^{-}$, respectively, for second or third generation leptoquarks. The limits are on the production cross section times $\beta^{2}$ where $\beta$ is the branching ratio into the final state searched for. In our model, $\beta \leq 1 / 2$ since $\rho$ can decay into either a charged lepton or neutrino with equal probability. Moreover, the branching ratio into muons is $\left|\lambda_{2}\right|^{2} /\left(\left|\lambda_{2}\right|^{2}+\left|\lambda_{e}\right|^{2}\right)$, and that into tau is $\left|\lambda_{3}\right|^{2} /\left(\left|\lambda_{2}\right|^{2}+\left|\lambda_{e}\right|^{2}\right)$, typically giving a further reduction in $\beta$. (Recall that LFV constraints on $\lambda_{1}$ make the branching into $e^{+} e^{-}$negligible in our model.)

The predicted cross sections and observed limits are shown in Fig. 10 for a model with $\Lambda_{H C}=100 \mathrm{GeV}$ and $m_{S} \ll \Lambda_{H C}$, which is a limiting case for lowering the masses of bound states containing $S$. We have assumed $\lambda_{3} \sim \lambda_{2}$ so that $\beta \cong 1 / 4$. The most stringent limits come from the search for final state muons, with production via the $g g \rightarrow \Psi \bar{\Psi}$ cross section (49). It requires the leptoquark mass to be $\gtrsim 1.1,1.2,1.26 \mathrm{TeV}$ for $N_{H C}=2,3,4$, hence $m_{\Psi} \gtrsim 670,590,470 \mathrm{GeV}$, respectively.

Other bound states not involving $S$ as a constituent, although they have potentially interesting signals, are too heavy to be produced at a significant level relative to current constraints from LHC. The composite lepton $F_{l}=S \phi^{*}$ arises from electroweak $q \bar{q} \rightarrow \phi \phi^{*}$ pair production, with parton-level cross section

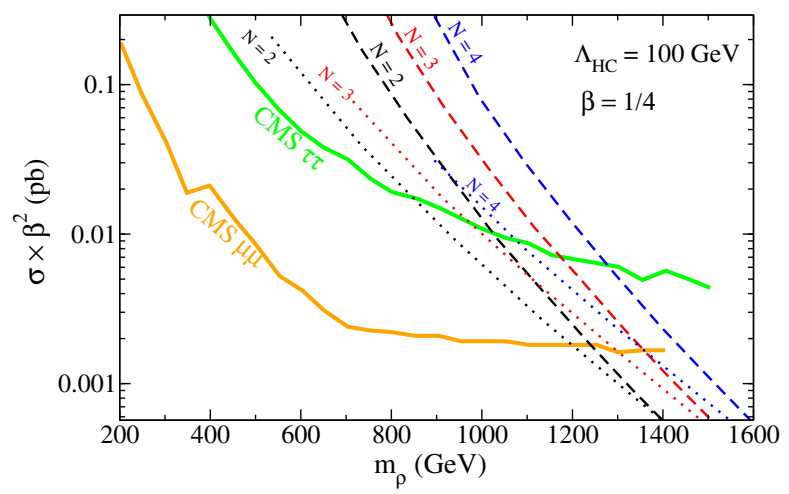

FIG. 10. Cross section for pair production of composite leptoquarks times branching ratio squared for decays into $\mu$ or $\tau$ plus jet $(j)$. Solid is CMS upper limit for $j j \mu \mu$ [120] or $j j \tau \tau$ [121] final states, dotted is prediction from $q \bar{q} \rightarrow \rho \rho^{*}$, dashed is prediction from $g g \rightarrow \rho \rho^{*}$, assuming low compositeness scale $\Lambda_{H C}=100 \mathrm{GeV}$ and massless $S$ constituents.

$$
\hat{\sigma}=\frac{\pi \alpha_{2}^{2} N_{H C}}{4} \frac{\hat{s}\left(1-4 M^{2} / \hat{s}\right)^{3 / 2}}{\left(\hat{s}-m_{W}^{2}\right)^{2}+m_{W}^{2} \Gamma_{W}^{2}} .
$$

in the simplifying approximation $g^{\prime} \ll g\left(\sin \theta_{W} \rightarrow 0\right)$. CMS has searched for excited muons and taus decaying to the normal state plus photon [122]. Figure 11 shows the limit on excited $\mu$ production, which is more stringent than that for excited $\tau$, versus the predicted cross sections (100\% branching of $F_{l} \rightarrow \mu \gamma$ is assumed). Again, we take the extreme choice with $\Lambda_{H C}=100 \mathrm{MeV}$ and $m_{S}=0$ to obtain light enough $m_{F_{l}}$ to fall within the currently probed mass range; larger values of $\Lambda_{H C}$ and $m_{S}$ are not excluded. Comparison with Fig. 10 shows that $F_{l}$ is excluded up to similar masses as the leptoquarks, $m_{F_{l}} \lesssim 1.3-1.5 \mathrm{TeV}$, depending upon $N_{H C}$.

The remaining mechanism to produce the lightest states is diagram (g) of Fig. 9, which has partonic cross section

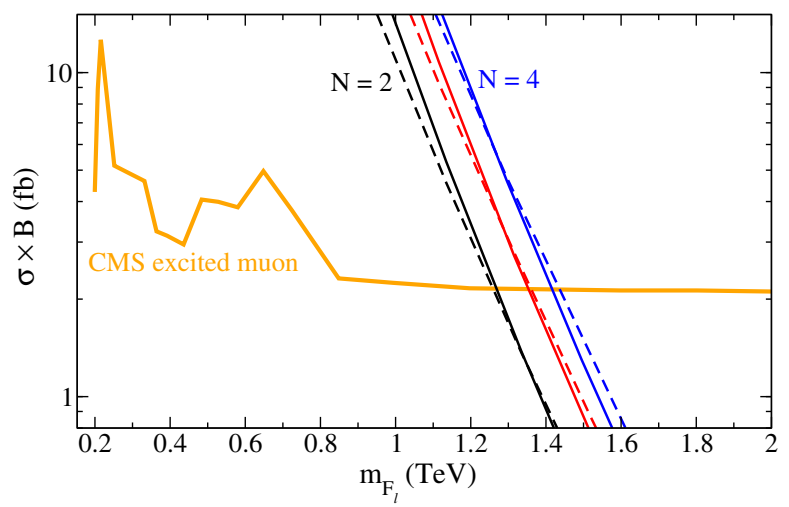

FIG. 11. Cross section for production of composite heavy leptons decaying to $\mu$ plus photon. Solid (orange) curve is CMS upper limit, dashed are predicted values from $F_{l}$ pair production, assuming low compositeness scale $\Lambda_{H C}=100 \mathrm{GeV}$ and massless $S$ constituents. Neighboring solid curves are from production of $F_{l}$-leptoquark pairs, Eq. (52). 


$$
\hat{\sigma}=N_{H C} \frac{\alpha_{s}\left|\tilde{\lambda}_{i}^{(\prime)}\right|^{2}}{24 \hat{s}} \sqrt{1-4 M^{2} / \hat{s}},
$$

neglecting the quark mass (a good approximation since the top quark PDF is very small). This produces the heavy lepton $F_{l}$ in conjunction with a leptoquark, and would be probed by the CMS search [122]. The predicted cross section (assuming 100\% $F_{l} \rightarrow \mu \gamma$ ) is also shown in Fig. 11 and happens to be close to that coming from $F_{l}$ pair production. Since the leptoquark is produced singly, it does not produce the same signal as for the existing searches that assume pair production and would require a new analysis, searching for the unusual final state of two leptons, a jet and a photon.

\section{SUMMARY AND CONCLUSIONS}

We have presented a particularly simple realization of composite leptoquarks to explain the anomalous $B$ decay ratios $R_{K^{(*)}}$, as well as providing a composite asymmetric dark matter candidate. The new ingredients are an $\mathrm{SU}\left(N_{H C}\right)$ confining gauge sector under which three types of matter fields $\Psi, S, \phi$ transform as fundamentals. Only two kinds of interactions with SM fermions are allowed, and these break the approximate flavor symmetries of the SM through the six couplings $\lambda_{i}$ to leptons and $\tilde{\lambda}_{i}$ to quarks. The leptoquark is the $\Psi \bar{S}$ vector bound state.

There is a limited region of parameter space allowed by FCNC constraints on meson-antimeson oscillations (mediated by the $\Psi \bar{\Psi}$ vector bound states), direct dark matter searches, and LHC searches for resonant dijets, which require the $\Psi$ and $\phi$ constituents to be near the TeV scale, and the new confinement scale $\Lambda_{H C}$ must be $\gtrsim 100 \mathrm{GeV}$. (Our benchmark model takes $\Lambda_{H C}=400 \mathrm{GeV}$.) The dark matter is a baryonlike bound state $\Sigma=S^{N_{H C}}$ with mass $\sim(1-5) \mathrm{TeV}$. If $N_{H C}$ is odd, $\Sigma$ can have a magnetic dipole moment that requires $m_{\Sigma}<2.5 \mathrm{TeV}$ to avoid direct detection.

For very light dark matter constituent and low confinement scale with $m_{S} \ll \Lambda_{H C} \sim 100$, the new bound states (leptoquarks and heavy lepton partners) are light enough $(\sim 1 \mathrm{TeV})$ to be pair-produced and significantly constrained by ATLAS or CMS searches for leptoquarks and excited leptons. The model also predicts production of the leptoquark and heavy lepton in association, leading to the unusual signal of two muons, a jet and a photon. The dark matter dipole moment is below direct detection constraints for small $m_{S}$.

The predictions are sensitive to nonperturbative aspects of confinement, especially bound state masses and wave functions. We have used a simple potential model that works reasonably well for known examples from QCD, but which is not necessarily reliable for the parameters of interest here, and neglects spin interactions between the fermionic consituents. Lattice studies of the masses and decay constants would allow for a useful improvement. Nevertheless, it seems likely that the kind of model presented here should give rise to other anomalous signals in FCNC, dark matter, or collider searches. It is encouraging that the experimental status of the $B$ decay anomalies should become more clear from future data at $\mathrm{LHCb}$ and Belle II during the next few years [123].

\section{ACKNOWLEDGMENTS}

I thank I. Brivio, J. Evans, B. Gavela, J. Jiang, D. London, J. Martin Camalich, Y. Kats, M. Redi, M. Trott for helpful discussions, and A. Urbano for pointing out Ref. [98]. This work was supported by NSERC (Natural Sciences and Engineering Research Council of Canada).

\section{APPENDIX A: POTENTIAL MODEL FOR BOUND STATES}

\section{Nonrelativistic constituents}

We first consider bound states where all the constituents are heavier than $\Lambda_{H C}$ and hence nonrelativistic. Following $[112,124]$, we estimate the size of hypercolor mesonic bound states using a variational method, with hydrogenlike Ansätze for the wave functions. The Coulomb-like contribution to the potential from hypergluon exchange is [125]

$$
V_{c}=-\frac{\alpha_{H C}}{2 r}\left(N_{H C}-\frac{1}{N_{H C}}\right) \equiv-c_{\alpha} \frac{\alpha_{H C}}{r}
$$

for a hyperquark-antiquark pair in the hypercolor singlet state. There is a linear confining potential

$$
V_{l}=\sigma r,
$$

where $\sigma \cong 2\left(N_{H C}-1\right) \Lambda_{H C}^{2}$ is the string tension and $\Lambda_{H C}$ is identified with the $\Lambda$ parameter of the 0 -flavor running coupling in the $\overline{\mathrm{MS}}$ scheme. The coefficient is deduced for the case of $N_{H C}=3$ from lattice studies [126] and its generalization to other values of $N_{H C}$ is inferred from large- $N$ scaling. The energy of an $s$-wave state with wave function

$$
\psi_{s}(r)=\frac{\mu_{*}^{3 / 2}}{\sqrt{8 \pi}} e^{-\mu_{*} r / 2}
$$

is

$$
E_{s}=m_{1}+m_{2}+\frac{\mu_{*}^{2}}{8 m_{r}}-\frac{c_{\alpha}}{2} \alpha_{H C} \mu_{*}+3 \frac{\sigma}{\mu_{*}},
$$

where $m_{r}$ is the reduced mass of the constituent particles, and $\alpha_{H C}$ implicitly depends on $\mu_{*}$ since it is the running coupling evaluated at that scale. We thus minimize the energy numerically. We use the 4-loop, 0-flavor expression for the running coupling given in [127,128]. For $\mu_{*}<$ $\Lambda_{H C}$ the perturbative coupling diverges and we cut it off 
at $\alpha_{H C}=2$. (Larger cutoffs lead to numerical artifacts in the minimization.)

This model gives reasonable results for $J / \psi$ and $\Upsilon$ using $m_{c}=1.3 \mathrm{GeV}, m_{b}=4.2 \mathrm{GeV}$ for the quark masses, and $\Lambda_{\mathrm{QCD}}=200 \mathrm{MeV}$. For these systems $\psi(0)$ is measurable through the electromagnetic decays via $\gamma^{*}$ to $e^{+} e^{-}$. For $J / \psi$ we find $\psi(0)^{2}=1 /(0.5 \mathrm{fm})^{3}$, close to the measured value $1 /(0.53 \mathrm{fm})^{3}$, while the mass is predicted to be $3.0 \mathrm{GeV}$, compared to the measured $3.1 \mathrm{GeV}$. The model predicts $\psi(0)_{\Upsilon}^{2} / \psi(0)_{J / \psi}^{2}=4.6$, while experimentally one finds 4.7, by comparing the decay rate formula [including the correction (42)] to the observed partial widths. The worst agreement is for $m_{\Upsilon}$, predicted to be $8.6 \mathrm{GeV}$ versus the measured value $9.5 \mathrm{GeV}$.

For $p$-wave states with

$$
\psi_{p}(r)=\frac{\mu_{*}^{5 / 2}}{\sqrt{96 \pi}} r e^{-\mu_{*} r / 2},
$$

we find the energy

$$
E_{p}=m_{1}+m_{2}+\frac{\mu_{*}^{2}}{8 m_{r}}-\frac{c_{\alpha}}{4} \alpha_{H C} \mu_{*}+5 \frac{\sigma}{\mu_{*}} .
$$

For baryons in an $s$-wave state, we take the Ansatz $\psi \sim e^{-\mu_{*}\left(r_{1}+r_{2}+\cdots\right)}$, and the energy is

$$
\begin{aligned}
E_{b}= & \sum_{i=1}^{N_{H C}}\left(m_{i}+\frac{\mu_{*}^{2}}{8 m_{i}}\right) \\
& +\frac{1}{2} N_{H C}\left(N_{H C}-1\right)\left(-\frac{5 c_{\alpha} \alpha_{H C} \mu_{*}}{16\left(N_{H C}-1\right)}+f \frac{35 \sigma}{8 \mu_{*}}\right)
\end{aligned}
$$

(note that the Coulomb attraction between $q q$ in an antisymmetric hypercolor state is $1 /\left(N_{H C}-1\right)$ times weaker than that between $q \bar{q}$ ). Comparison to the nucleon of QCD motivates the correction factor $f=0.065$, indicating that the string tension is much smaller between $q q$ than $q \bar{q}$ states.

\section{Heavy-light or relativistic systems}

In the case where $m_{S}<\Lambda_{H C}$, one or more constituents is relativistic. Then, in the center of mass system of a heavylight meson we have mass plus kinetic energy $m_{1}+p^{2} /$ $\left(2 m_{1}\right)+\sqrt{p^{2}+m_{2}}$ in the Hamiltonian [129]. Still using the wave function Ansatz $\psi \sim e^{-\mu_{*} r / 2}$ for $s$-wave states, the expectation value of $\sqrt{p^{2}+m^{2}}$ can be found by Fourier transforming $\psi$ and evaluating the integral in the momentum eigenstate basis. The result is a complicated analytic function that can be fit to the simpler form

$$
\left\langle\sqrt{p^{2}+m^{2}}\right\rangle \cong \mu_{*}\left(0.84+0.52\left(\frac{m}{\mu_{*}}\right)^{1.6}\right) .
$$

[At $m=0$ the exact result is $8 \mu_{*} /(3 \pi)$.] We find that this approximation is good to better than $1 \%$ for $m<\mu_{*}$. The kinetic energy in (A4) and (A6) is then replaced by

$$
m_{1}+\frac{\mu_{*}^{2}}{8 m_{1}}+\left\langle\sqrt{p^{2}+m_{2}^{2}}\right\rangle .
$$

For baryons with some constituents light, the appropriate replacement is obvious. The coupling $\alpha_{H C}$ should be evaluated taking into account running with the appropriate number of light constituents.

\section{APPENDIX B: TRANSITION MOMENTS FROM COMPOSITENESS}

Here we semiquantitatively estimate the transition magnetic moment interactions between heavy composite fermions $F_{R}$ and their elementary SM counterparts, $f_{R}$, following the same formalism used by Ref. [98] for radiative decays of the $Z$ boson to quarkonium bound states. The formalism is appropriate for nonrelativistic systems, which is not a very good approximation in our case since we prefer the constituent masses and confinement scale to be of the same order. Hopefully it gives a reasonable estimate, which would require a dedicated lattice study to improve upon.

The amplitude for $F \rightarrow f_{i} \gamma$ can be written as

$$
A\left(F \rightarrow f_{i} \gamma\right)=-i \stackrel{(\sim)}{\lambda_{i}} \bar{u}_{f_{i}} \int \frac{d^{4} q}{(2 \pi)^{4}} \mathcal{O}_{\gamma}(q, k, Q) \chi(Q, k),
$$

where the momenta are indicated in Fig. 3. $Q$ is the momentum of they decaying heavy $F, k$ is that of the photon, $q$ is the relative momentum between the heavy fermion constituents and $\chi(Q, q)$ is the Bethe-Salpeter wave function for the bound state. The operator $\mathcal{O}_{\gamma}$ comes from the part of the diagram that is introduced by insertion of the photon vertex, which depends upon what kind of particle $F(f)$ is. For downlike quarks, the photon attaches to both $\Psi$ and $\phi^{ \pm}$; for uplike quarks it attaches only to $\Psi$ (since $\phi^{0}$ is the other constituent), while for leptons it attaches only to $\phi^{ \pm}$since $S$ is neutral.

For simplicity we take the constituent masses to be equal so that, in the absence of exchanged momenta, each carries half of the momentum $Q^{\mu}$ of the bound state. The general form of $\mathcal{O}$ can then be written as

$$
\begin{aligned}
\mathcal{O}_{\gamma}= & -i e q_{\phi} \frac{i(-Q+2 q+k)^{\mu}}{(-Q / 2+q+k)^{2}-m_{\phi}^{2}} P_{R} \\
& -i e q_{\psi(S)} \frac{i}{\Phi / 2+\not 1-\not k-m_{\psi(S)}} \gamma^{\mu} P_{R},
\end{aligned}
$$

where $P_{R}$ projects onto right-handed chirality. The spinor wave function takes the form 
$\chi(Q, q)=\frac{2 \pi}{\sqrt{2 \mu}} \delta\left(q_{0}-\frac{\vec{q}^{2}}{2 \mu}\right) u_{\psi(S)}(Q / 2+q) \tilde{\psi}(q)$,

where $\mu$ is the reduced mass of the bound state constituents, $u_{\psi(S)}$ is the Dirac spinor for the fermionic member, and $\tilde{\psi}$ is the Fourier transform of the spatial wave function.

For nonrelativistic systems, the wave function is strongly peaked at small $\vec{q}$ and it is a good approximation to set $q=0$ in $\mathcal{O}_{\gamma}$. Then the factor $\int d^{3} q /(2 \pi)^{3} \tilde{\psi}(q)=\psi(0)$, the spatial wave function evaluated at the origin, and the amplitude becomes

$A\left(F \rightarrow f_{i} \gamma\right)=-i \frac{(\sim)}{\hat{\lambda}_{i} \psi(0)} \sqrt{2 \mu} \bar{u}_{f_{i}} \mathcal{O}_{\gamma}(0, k, Q) P_{R} u_{\psi(S)}$.

It is pertinent to compare this to the corresponding amplitude with no photon, which is just the mass mixing amplitude,

$A\left(F \rightarrow f_{i}\right)=-i \frac{\stackrel{(\sim)}{\lambda_{i} \psi(0)}}{\sqrt{2 \mu}} \bar{u}_{f_{i}} P_{R} u_{\psi(S)}=-i \mu_{f}^{i} \bar{u}_{f_{i}} P_{R} u_{\psi(S)}$.

This allows us to infer that the transition moment is related to the mass mixing in a definite way.

For leptons, the decay amplitude from diagram (a) of Fig. 3 is

$$
\begin{aligned}
A\left(F_{\ell} \rightarrow \ell_{i} \gamma\right)= & -i e \mu_{\ell}^{i} \bar{u}_{\ell_{i L}} \frac{(Q-k)^{\mu}}{\frac{1}{4} m_{F_{\ell}}^{2}+m_{\phi}^{2}} u_{S_{R}} \\
& \rightarrow-i e \frac{\mu_{\ell}^{i}}{m_{F_{\ell}}^{2}} \bar{u}_{\ell_{i L}} i \sigma^{\mu \nu} k_{\nu} u_{S_{R}},
\end{aligned}
$$

where in the second line we used the Gordon identity

$\left(p+p^{\prime}\right)^{\mu} \bar{u}_{L}^{\prime} u_{R}=m^{\prime} \bar{u}_{R}^{\prime} \gamma^{\mu} u_{R}+m \bar{u}_{L}^{\prime} \gamma^{\mu} u_{L}+\bar{u}_{L}^{\prime} i \sigma^{\mu \nu} k_{\nu} u_{R}$

and took $m_{\phi} \simeq m_{F_{\ell}} / 2$. The extra terms going as $\gamma^{\mu}$ in the Gordon identity are canceled by diagrams (c) and (d) of Fig. 3, which serves as a check on the sign. We ignored terms going as $k^{\mu}$ that do not contribute to the amplitude because of the transversality of the photon polarization vector.

Similarly for up- and down-type quarks, respectively, we obtain

$$
\begin{aligned}
& A\left(F_{u} \rightarrow u_{i} \gamma\right)=i \frac{2 e}{3} \frac{\mu_{u}^{\prime i}}{m_{F_{u}}^{2}} \bar{u}_{u_{i L}} i \sigma^{\mu \nu} k_{\nu} u_{\Psi_{R}}, \\
& A\left(F_{d} \rightarrow d_{i} \gamma\right)=-i \frac{e}{3} \frac{\mu_{d}^{i}}{m_{F_{d}}^{2}} \bar{u}_{d_{i L}} i \sigma^{\mu \nu} k_{\nu} u_{\Psi_{R}},
\end{aligned}
$$

where $\mu_{u}^{\prime i}=\tilde{\lambda}_{i}^{\prime} \Lambda_{H C}$ with $\tilde{\lambda}_{i}^{\prime}$ given in Eq. (5), and $\mu_{d}^{i}=\tilde{\lambda}_{i} \Lambda_{H C}$. After rotating the heavy and light fields to eliminate mass mixing, these lead to magnetic moments involving just the light states.

\section{APPENDIX C: DM DIPOLE MOMENT}

We give details for the one-loop contribution to the magnetic dipole moment of the elementary dark matter particle $S$, with diagrams shown in Fig. 12. They turn out to be infrared convergent in the limit $m_{\mu} \rightarrow 0$ for the internal muon, hence we make this simplification. The 1PI diagrams (a) and (b) in Fig. 12 respectively give

$$
\begin{aligned}
& e\left|\lambda_{2}\right|^{2} 2 \int_{0}^{1} d x \int_{0}^{1-x} d y \int \frac{d^{4} l}{(2 \pi)^{4}} \bar{u}_{p^{\prime}} \\
& \times \frac{\left(l+p^{\prime}\right) \gamma^{\mu}(\not+\not p) P_{R}}{\left[\left(l+x p^{\prime}+y p\right)^{2}-M_{a}^{2}\right]^{3}} u_{p}, \\
& e\left|\lambda_{2}\right|^{2} 2 \int_{0}^{1} d x \int_{0}^{1-x} d y \int \frac{d^{4} l}{(2 \pi)^{4}} \bar{u}_{p^{\prime}} \\
& \times \frac{\left(2 l-p-p^{\prime}\right)^{\mu} \mid P_{R}}{\left[\left(l-x p-y p^{\prime}\right)^{2}-M_{a}^{2}\right]^{3}} u_{p},
\end{aligned}
$$

where, defining $u=x+y$,

$$
\begin{aligned}
& M_{a}^{2}=(1-u)\left[m_{\phi}^{2}-u m_{S}^{2}\right], \\
& M_{b}^{2}=u\left[m_{\phi}^{2}-(1-u) m_{S}^{2}\right] .
\end{aligned}
$$

As usual, one shifts the loop momentum variable to simplify the denominators. Only the finite parts contribute to the dipole operator. To extract the dipole moment, one makes the replacements

$$
\begin{aligned}
& P_{L} \not \gamma^{\mu} \rightarrow P_{L}(\not p+q) \gamma^{\mu} \rightarrow\left(P_{R} m_{S}+P_{L} q\right) \gamma^{\mu}, \\
& \gamma^{\mu} \not \not^{\prime} P_{R} \rightarrow \gamma^{\mu}(\not p-q) P_{R} \rightarrow \gamma^{\mu}\left(m_{S} P_{L}-q P_{R}\right)
\end{aligned}
$$

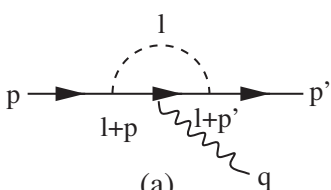

(a)

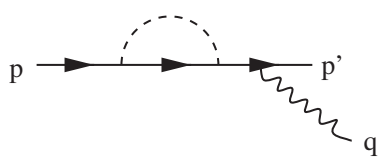

(c)

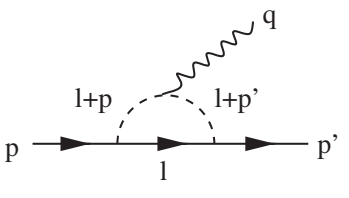

(b)

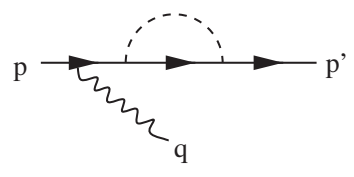

(d)
FIG. 12. Contributions to elementary dark matter magnetic dipole moment. 
in the diagram in Fig. 12(a), using the Dirac equation (and similarly letting $\left.\not p^{\prime} \gamma^{\mu} \rightarrow m_{S} \gamma^{\mu}, \gamma^{\mu} \not \supset \rightarrow m_{S} \gamma^{\mu}\right)$, and keeping the terms linear in $\not$. Both of these can be put into the form $\gamma^{\mu} \not$ up to terms going as $q^{\mu}$, which give a vanishing contribution when contracted with the external photon polarization vector. These are directly related to the magnetic moment since $-i \sigma_{\mu \nu} q^{\nu} \rightarrow \gamma^{\mu} \mathscr{Q}$ in this way.

In the diagram in Fig. 12(b), we again use the Dirac equation to rewrite $\not p P_{R} \rightarrow m_{S} P_{L}, P_{L} \not \not^{\prime} \rightarrow m_{S} P_{R}$, and reexpress $p^{\mu}, p^{\prime \mu}$ as linear combinations of $\left(p+p^{\prime}\right)^{\mu}$ and $q^{\mu}$, of which only the former contribute to the amplitude. Using the Gordon identity, $\left(p+p^{\prime}\right)^{\mu} \rightarrow i \sigma_{\mu \nu} q^{\nu} \rightarrow-\gamma^{\mu} \not$. We find that both diagrams (a) and (b) in Fig. 12 contribute with the same sign. A check on this relative sign is provided by gauge invariance: the coupling $\bar{S} \gamma^{\mu} S A_{\mu}$ is forbidden, and must not be generated by loops. The divergent parts of diagrams (a) and (b) in Fig. 12 contribute with the same magnitude and sign to the vector current, and these cancel the contributions from Figs. 12(c) and 12(d). Such a cancellation would not occur if Figs. 12(a) and 12(b) came with opposite signs.

Defining $R=m_{S}^{2} / m_{\phi}^{2}$, the final expression for the magnetic moment (which is the coefficient of $\bar{u}_{p^{\prime}} \gamma^{\mu} \not u_{p}$ ) is

$$
\mu_{S}=\frac{e\left|\lambda_{2}\right|^{2}}{32 \pi^{2} m_{\phi}^{2}}\left(f_{a}(R)+f_{b}(R)\right)
$$

with

$$
\begin{aligned}
& f_{a}(R)=\int_{0}^{1} d u \frac{u^{2}}{1-R u} \\
& f_{b}(R)=\int_{0}^{1} d u \frac{u(1-u)}{1-R+R u} .
\end{aligned}
$$

In the limit of light dark matter, $f_{a}(0)+f_{b}(0)=1 / 2$.
[1] R. Aaij et al. (LHCb Collaboration), Test of Lepton Universality Using $B^{+} \rightarrow K^{+} \ell^{+} \ell^{-}$Decays, Phys. Rev. Lett. 113, 151601 (2014).

[2] R. Aaij et al. (LHCb Collaboration), Test of lepton universality with $B^{0} \rightarrow K^{* 0} \ell^{+} \ell^{-}$decays, J. High Energy Phys. 08 (2017) 055.

[3] L. S. Geng, B. Grinstein, S. Jäger, J. Martin Camalich, X. L. Ren, and R.X. Shi, Towards the discovery of new physics with lepton-universality ratios of $b \rightarrow s \ell \ell$ decays, Phys. Rev. D 96, 093006 (2017).

[4] G. D’Amico, M. Nardecchia, P. Panci, F. Sannino, A. Strumia, R. Torre, and A. Urbano, Flavour anomalies after the $R_{K^{*}}$ measurement, J. High Energy Phys. 09 (2017) 010.

[5] W. Altmannshofer, P. Stangl, and D. M. Straub, Interpreting hints for lepton flavor universality violation, Phys. Rev. D 96, 055008 (2017).

[6] B. Capdevila, A. Crivellin, S. Descotes-Genon, J. Matias, and J. Virto, Patterns of New Physics in $b \rightarrow s \ell^{+} \ell^{-}$ transitions in the light of recent data, arXiv:1704.05340.

[7] A. Celis, J. Fuentes-Martin, A. Vicente, and J. Virto, Gauge-invariant implications of the LHCb measurements on lepton-flavor nonuniversality, Phys. Rev. D 96, 035026 (2017).

[8] M. Ciuchini, A. M. Coutinho, M. Fedele, E. Franco, A. Paul, L. Silvestrini, and M. Valli, On flavourful easter eggs for New Physics hunger and lepton flavour universality violation, Eur. Phys. J. C 77, 688 (2017).

[9] D. Bardhan, P. Byakti, and D. Ghosh, Role of Tensor operators in $R_{K}$ and $R_{K^{*}}$, Phys. Lett. B 773, 505 (2017).

[10] S. Neshatpour, V. G. Chobanova, T. Hurth, F. Mahmoudi, and D. Martinez Santos, Direct comparison of global fits to the $B \rightarrow K^{*} \mu^{+} \mu^{-}$data assuming hadronic corrections or new physics, arXiv:1705.10730.
[11] A. K. Alok, D. Kumar, J. Kumar, and R. Sharma, Lepton flavor non-universality in the B-sector: A global analysis of various new physics models, arXiv:1704.07347.

[12] A. K. Alok, B. Bhattacharya, A. Datta, D. Kumar, J. Kumar, and D. London, New Physics in $b \rightarrow s \mu^{+} \mu^{-}$ after the Measurement of $R_{K^{*}}$, Phys. Rev. D 96, 095009 (2017).

[13] D. Choudhury, A. Kundu, R. Mandal, and R. Sinha, Minimal Unified Resolution to $R_{K^{(*)}}$ and $R\left(D^{(*)}\right)$ Anomalies with Lepton Mixing, Phys. Rev. Lett. 119, 151801 (2017).

[14] B. Gripaios, M. Nardecchia, and S. A. Renner, Linear flavour violation and anomalies in B physics, J. High Energy Phys. 06 (2016) 083.

[15] M. Bauer and M. Neubert, Minimal Leptoquark Explanation for the $\mathrm{R}_{D^{(*)}}, \mathrm{R}_{K}$, and $(g-2)_{g}$ Anomalies, Phys. Rev. Lett. 116, 141802 (2016).

[16] P. Arnan, L. Hofer, F. Mescia, and A. Crivellin, Loop effects of heavy new scalars and fermions in $b \rightarrow s \mu^{+} \mu^{-}$, J. High Energy Phys. 04 (2017) 043.

[17] J. F. Kamenik, Y. Soreq, and J. Zupan, Lepton flavor universality violation without new sources of quark flavor violation, arXiv:1704.06005 [Phys. Rev. D (to be published)].

[18] D. Das, C. Hati, G. Kumar, and N. Mahajan, Scrutinizing $R$-parity violating interactions in light of $R_{K^{(*)}}$ data, Phys. Rev. D 96, 095033 (2017).

[19] J. Kawamura, S. Okawa, and Y. Omura, Impact of the $b \rightarrow$ sll anomalies on dark matter physics, Phys. Rev. D 96, 075041 (2017).

[20] X. G. He and G. Valencia, Are the B-anomalies evidence for heavy neutrinos?, arXiv:1706.07570. 
[21] R. Gauld, F. Goertz, and U. Haisch, On minimal $Z^{\prime}$ explanations of the $B \rightarrow K^{*} \mu^{+} \mu^{-}$anomaly, Phys. Rev. D 89, 015005 (2014).

[22] R. Gauld, F. Goertz, and U. Haisch, An explicit Z'-boson explanation of the $B \rightarrow K^{*} \mu^{+} \mu^{-}$anomaly, J. High Energy Phys. 01 (2014) 069.

[23] A. J. Buras, F. De Fazio, and J. Girrbach, 331 models facing new $b \rightarrow s \mu^{+} \mu^{-}$data, J. High Energy Phys. 02 (2014) 112.

[24] W. Altmannshofer, S. Gori, M. Pospelov, and I. Yavin, Quark flavor transitions in $L_{\mu}-L_{\tau}$ models, Phys. Rev. D 89, 095033 (2014).

[25] A. Crivellin, G. D'Ambrosio, and J. Heeck, Addressing the LHC flavor anomalies with horizontal gauge symmetries, Phys. Rev. D 91, 075006 (2015).

[26] D. Aristizabal Sierra, F. Staub, and A. Vicente, Shedding light on the $b \rightarrow s$ anomalies with a dark sector, Phys. Rev. D 92, 015001 (2015).

[27] A. Crivellin, L. Hofer, J. Matias, U. Nierste, S. Pokorski, and J. Rosiek, Lepton-flavour violating $B$ decays in generic $Z^{\prime}$ models, Phys. Rev. D 92, 054013 (2015).

[28] A. Celis, J. Fuentes-Martin, M. Jung, and H. Serodio, Family nonuniversal $Z^{\prime}$ models with protected flavorchanging interactions, Phys. Rev. D 92, 015007 (2015).

[29] A. Carmona and F. Goertz, Lepton Flavor and Nonuniversality from Minimal Composite Higgs Setups, Phys. Rev. Lett. 116, 251801 (2016).

[30] K. Fuyuto, W. S. Hou, and M. Kohda, $Z^{\prime}$-induced FCNC decays of top, beauty, and strange quarks, Phys. Rev. D 93, 054021 (2016).

[31] C. W. Chiang, X. G. He, and G. Valencia, $Z^{\prime}$ model for $b \rightarrow s \ell \bar{l}$ flavor anomalies, Phys. Rev. D 93, 074003 (2016).

[32] C. S. Kim, X. B. Yuan, and Y. J. Zheng, Constraints on a $Z^{\prime}$ boson within minimal flavor violation, Phys. Rev. D 93, 095009 (2016).

[33] S. M. Boucenna, A. Celis, J. Fuentes-Martin, A. Vicente, and J. Virto, Non-Abelian gauge extensions for B-decay anomalies, Phys. Lett. B 760, 214 (2016).

[34] K. Cheung, W. Y. Keung, and P. Y. Tseng, Lepton-flavorviolating $Z^{\prime}$ using the electron-muon channel at the LHC, Phys. Rev. D 94, 075006 (2016).

[35] S. M. Boucenna, A. Celis, J. Fuentes-Martin, A. Vicente, and J. Virto, Phenomenology of an $S U(2) \times S U(2) \times$ $U(1)$ model with lepton-flavour non-universality, J. High Energy Phys. 12 (2016) 059.

[36] A. Crivellin, J. Fuentes-Martin, A. Greljo, and G. Isidori, Lepton flavor non-universality in B decays from dynamical Yukawas, Phys. Lett. B 766, 77 (2017).

[37] I. Garcia Garcia, LHCb anomalies from a natural perspective, J. High Energy Phys. 03 (2017) 040.

[38] A. Datta, J. Liao, and D. Marfatia, A light $Z^{\prime}$ for the $R_{K}$ puzzle and nonstandard neutrino interactions, Phys. Lett. B 768, 265 (2017).

[39] P. Ko, Y. Omura, Y. Shigekami, and C. Yu, The LHCb anomaly and $B$ physics in flavored $Z^{\prime}$ models with flavored Higgs doublets, Phys. Rev. D 95, 115040 (2017).

[40] R. Alonso, P. Cox, C. Han, and T. T. Yanagida, Anomalyfree local horizontal symmetry and anomaly-full rare B-decays, Phys. Rev. D 96, 071701 (2017).
[41] C. Bonilla, T. Modak, R. Srivastava, and J. W. F. Valle, $U(1)_{B_{3}-3 L_{\mu}}$ gauge symmetry as the simplest description of $b \rightarrow s$ anomalies, arXiv:1705.00915.

[42] R. Alonso, P. Cox, C. Han, and T. T. Yanagida, Flavoured $B-L$ local symmetry and anomalous rare $B$ decays, Phys. Lett. B 774, 643 (2017).

[43] J. Ellis, M. Fairbairn, and P. Tunney, Anomaly-Free Models for Flavour Anomalies, arXiv:1705.03447.

[44] D. Ghosh, Explaining the $R_{K}$ and $R_{K^{*}}$ anomalies, Eur. Phys. J. C 77, 694 (2017).

[45] Y. Tang and Y. L. Wu, Flavor Non-universality Gauge Interactions and Anomalies in B-Meson Decays, arXiv: 1705.05643 .

[46] C. W. Chiang, X. G. He, J. Tandean, and X. B. Yuan, $R_{K^{(*)}}$ and related $b \rightarrow s \ell \bar{l}$ anomalies in minimal flavor violation framework with $Z^{\prime}$ boson, Phys. Rev. D 96, 115022 (2017).

[47] R. S. Chivukula, J. Isaacson, K. A. Mohan, D. Sengupta, and E. H. Simmons, $R_{K}$ anomalies and simplified limits on $Z^{\prime}$ models at the LHC, Phys. Rev. D 96, 075012 (2017).

[48] S. F. King, Flavourful $Z^{\prime}$ models for $R_{K^{(*)}}$, J. High Energy Phys. 08 (2017) 019.

[49] J. M. Cline and J. Martin Camalich, $B$ decay anomalies from non-Abelian local horizontal symmetry, Phys. Rev. D 96, 055036 (2017).

[50] C. H. Chen and T. Nomura, Penguin $b \rightarrow s \ell^{\prime+} \ell^{\prime-}$ and $B$-meson anomalies in a gauged $L_{\mu}-L_{\tau}$, arXiv: 1707 . 03249.

[51] S. Baek, Dark matter contribution to $b \rightarrow s \mu^{+} \mu^{-}$anomaly in local $U(1)_{L_{\mu}-L_{\tau}}$ model, arXiv:1707.04573.

[52] L. Bian, S. M. Choi, Y. J. Kang, and H. M. Lee, A minimal flavored $U(1)^{\prime}$ for $B$-meson anomalies, Phys. Rev. D 96, 075038 (2017).

[53] M. Dalchenko, B. Dutta, R. Eusebi, P. Huang, T. Kamon, and D. Rathjens, Bottom-quark Fusion Processes at the LHC for Probing $Z^{\prime}$ Models and B-meson Decay Anomalies, arXiv:1707.07016.

[54] E. Megias, M. Quiros, and L. Salas, Flavor anomalies from warped space, arXiv:1709.05100.

[55] I. Doršner, S. Fajfer, A. Greljo, J. F. Kamenik, and N. Košnik, Physics of leptoquarks in precision experiments and at particle colliders, Phys. Rep. 641, 1 (2016).

[56] G. Hiller and M. Schmaltz, $R_{K}$ and future $b \rightarrow s \ell \ell$ physics beyond the standard model opportunities, Phys. Rev. D 90 , 054014 (2014).

[57] B. Gripaios, M. Nardecchia, and S. A. Renner, Composite leptoquarks and anomalies in $B$-meson decays, J. High Energy Phys. 05 (2015) 006.

[58] B. Allanach, A. Alves, F. S. Queiroz, K. Sinha, and A. Strumia, Interpreting the CMS $\ell^{+} \ell^{-} j j E /_{\mathrm{T}}$ excess with a leptoquark model, Phys. Rev. D 92, 055023 (2015).

[59] R. Alonso, B. Grinstein, and J. Martin Camalich, Lepton universality violation and lepton flavor conservation in B-meson decays, J. High Energy Phys. 10 (2015) 184.

[60] S. Sahoo and R. Mohanta, Lepton flavor violating B meson decays via a scalar leptoquark, Phys. Rev. D 93, 114001 (2016).

[61] G. Kumar, Constraints on a scalar leptoquark from the kaon sector, Phys. Rev. D 94, 014022 (2016). 
[62] D. Das, C. Hati, G. Kumar, and N. Mahajan, Towards a unified explanation of $R_{D^{(*)}}, R_{K}$ and $(g-2)_{\mu}$ anomalies in a left-right model with leptoquarks, Phys. Rev. D 94, 055034 (2016).

[63] X. Q. Li, Y. D. Yang, and X. Zhang, Revisiting the one leptoquark solution to the $R\left(\mathrm{D}^{(*)}\right)$ anomalies and its phenomenological implications, J. High Energy Phys. 08 (2016) 054.

[64] C. H. Chen, T. Nomura, and H. Okada, Explanation of $B \rightarrow K^{(*)} \ell^{+} \ell^{-}$and muon $g-2$, and implications at the LHC, Phys. Rev. D 94, 115005 (2016).

[65] D. Bečirević, S. Fajfer, N. Košnik, and O. Sumensari, Leptoquark model to explain the $B$-physics anomalies, $R_{K}$ and $R_{D}$, Phys. Rev. D 94, 115021 (2016).

[66] D. Bečirević, N. Košnik, O. Sumensari, and R. Zukanovich Funchal, Palatable leptoquark scenarios for lepton flavor violation in exclusive $b \rightarrow s \ell_{1} \ell_{2}$ modes, J. High Energy Phys. 11 (2016) 035.

[67] N. Mileo, A. de la Puente, and A. Szynkman, Implications of an electroweak triplet scalar leptoquark on the ultra-high energy neutrino events at IceCube, J. High Energy Phys. 11 (2016) 124.

[68] G. Hiller, D. Loose, and K. Schönwald, Leptoquark flavor patterns \& B decay anomalies, J. High Energy Phys. 12 (2016) 027.

[69] S. Sahoo, R. Mohanta, and A. K. Giri, Explaining the $R_{K}$ and $R_{D^{(*)}}$ anomalies with vector leptoquarks, Phys. Rev. D 95, 035027 (2017).

[70] O. Popov and G. A. White, One Leptoquark to unify them? Neutrino masses and unification in the light of $(g-2)_{\mu}$, $R_{D^{(\star)}}$ and $R_{K}$ anomalies, Nucl. Phys. B923, 324 (2017).

[71] R. Barbieri, C. W. Murphy, and F. Senia, B-decay anomalies in a composite leptoquark model, Eur. Phys. J. C 77, 8 (2017).

[72] J. M. Cline, J. M. Cornell, D. London, and R. Watanabe, Hidden sector explanation of $B$-decay and cosmic ray anomalies, Phys. Rev. D 95, 095015 (2017).

[73] C. H. Chen, T. Nomura, and H. Okada, Excesses of muon $g-2, R_{D^{(*)}}$, and $R_{K}$ in a leptoquark model, Phys. Lett. B 774, 456 (2017).

[74] A. Crivellin, D. Müller, and T. Ota, Simultaneous explanation of $R\left(D^{(*)}\right)$ and $b \rightarrow s \mu^{+} \mu^{-}$: The last scalar leptoquarks standing, J. High Energy Phys. 09 (2017) 040.

[75] G. Hiller and I. Nisandzic, $R_{K}$ and $R_{K^{*}}$ beyond the Standard Model, Phys. Rev. D 96, 035003 (2017).

[76] Y. Cai, J. Gargalionis, M. A. Schmidt, and R. R. Volkas, Reconsidering the One Leptoquark solution: Flavor anomalies and neutrino mass, J. High Energy Phys. 10 (2017) 047.

[77] D. Bečirević and O. Sumensari, A leptoquark model to accommodate $R_{K}^{\text {exp }}$, J. High Energy Phys. 08 (2017) 104.

[78] S. Matsuzaki, K. Nishiwaki, and R. Watanabe, Phenomenology of flavorful composite vector bosons in light of $B$ anomalies, J. High Energy Phys. 08 (2017) 145.

[79] B. Chauhan, B. Kindra, and A. Narang, A Leptoquark explanation for $(g-2)_{\mu}, R_{K}, R_{K^{\star}}$ and, IceCube $\mathrm{PeV}$ events, arXiv:1706.04598.

[80] W. Altmannshofer, P.S. B. Dev, and A. Soni, $R_{D^{(*)}}$ anomaly: A possible hint for natural supersymmetry with $R$-parity violation, Phys. Rev. D 96, 095010 (2017).
[81] D. Buttazzo, A. Greljo, G. Isidori, and D. Marzocca, B-physics anomalies: A guide to combined explanations, J. High Energy Phys. 11 (2017) 044.

[82] A. Crivellin, D. Mueller, A. Signer, and Y. Ulrich, Correlating Lepton Flavour (Universality) Violation in $B$ Decays with $\mu \rightarrow$ er using Leptoquarks, arXiv:1706 .08511 .

[83] S. Y. Guo, Z. L. Han, B. Li, Y. Liao, and X. D. Ma, Interpreting the $R_{K^{(*)}}$ Anomaly in the Colored Zee-Babu Model, arXiv:1707.00522.

[84] D. Aloni, A. Dery, C. Frugiuele, and Y. Nir, Testing minimal flavor violation in leptoquark models of the $R_{K^{(*)}}$ anomaly, J. High Energy Phys. 11 (2017) 109.

[85] N. Assad, B. Fornal, and B. Grinstein, Baryon Number and Lepton Universality Violation in Leptoquark and Diquark Models, arXiv:1708.06350.

[86] L. Di Luzio, A. Greljo, and M. Nardecchia, Gauge leptoquark as the origin of B-physics anomalies, Phys. Rev. D 96, 115011 (2017).

[87] L. Calibbi, A. Crivellin, and T. Li, A model of vector leptoquarks in view of the $B$-physics anomalies, arXiv: 1709.00692.

[88] C. Kilic, T. Okui, and R. Sundrum, Vectorlike confinement at the LHC, J. High Energy Phys. 02 (2010) 018.

[89] J. Kang and M. A. Luty, Macroscopic strings and quirks at colliders, J. High Energy Phys. 11 (2009) 065.

[90] C. C. Nishi, Simple derivation of general Fierz-like identities, Am. J. Phys. 73, 1160 (2005).

[91] M. Bona et al. (UTfit Collaboration), Model-independent constraints on $\Delta F=2$ operators and the scale of new physics, J. High Energy Phys. 03 (2008) 049.

[92] M. Bona (UTfit Collaboration), Proc. Sci., ICHEP2016 (2016) 149.

[93] Z. Ligeti and F. Sala, A new look at the theory uncertainty of $\epsilon_{K}$, J. High Energy Phys. 09 (2016) 083; Erratum to: A new look at the theory uncertainty of $\epsilon_{K}$, J. High Energy Phys. 02 (2017) 140(E).

[94] O. Gedalia, Y. Grossman, Y. Nir, and G. Perez, Lessons from recent measurements of D0-anti-D0 Mixing, Phys. Rev. D 80, 055024 (2009).

[95] UTfit Collaboration, http://www.utfit.org/UTfit/Results Summer2016NP.

[96] G. D’Ambrosio, G. F. Giudice, G. Isidori, and A. Strumia, Minimal flavor violation: An effective field theory approach, Nucl. Phys. B645, 155 (2002).

[97] J. M. Cline, W. Huang, and G. D. Moore, Challenges for models with composite states, Phys. Rev. D 94, 055029 (2016).

[98] B. Guberina, J. H. Kuhn, R. D. Peccei, and R. Ruckl, Rare decays of the Z0, Nucl. Phys. B174, 317 (1980).

[99] P. G. Esposito, Constraints on composite models from leptonic flavor changing decays, Nuovo Cimento Soc. Ital. Fis. 92A, 328 (1986).

[100] S. Descotes-Genon, L. Hofer, J. Matias, and J. Virto, Global analysis of $b \rightarrow s \ell l$ anomalies, J. High Energy Phys. 06 (2016) 092.

[101] J. M. Cline, Scalar doublet models confront $\tau$ and $b$ anomalies, Phys. Rev. D 93, 075017 (2016). 
[102] C. Giunti and A. Studenikin, Neutrino electromagnetic interactions: a window to new physics, Rev. Mod. Phys. 87, 531 (2015).

[103] See the review of the muon anomalous magnetic moment in C. Patrignani et al. (Particle Data Group), Review of Particle Physics, Chin. Phys. C 40, 100001 (2016).

[104] M. E. Peskin and T. Takeuchi, Estimation of oblique electroweak corrections, Phys. Rev. D 46, 381 (1992).

[105] A. Mitridate, M. Redi, J. Smirnov, and A. Strumia, Dark Matter as a weakly coupled dark baryon, J. High Energy Phys. 10 (2017) 210.

[106] X. Cui et al. (PandaX-II Collaboration), Dark Matter Results from 54-Ton-Day Exposure of PandaX-II Experiment, Phys. Rev. Lett. 119, 181302 (2017).

[107] T. Banks, J. F. Fortin, and S. Thomas, Direct Detection of Dark Matter Electromagnetic Dipole Moments, arXiv: 1007.5515.

[108] E. Aprile et al. (XENON100 Collaboration), First Dark Matter Results from the XENON100 Experiment, Phys. Rev. Lett. 105, 131302 (2010).

[109] Y. Chen et al., Glueball spectrum and matrix elements on anisotropic lattices, Phys. Rev. D 73, 014516 (2006).

[110] C. Kilic, T. Okui, and R. Sundrum, Colored resonances at the Tevatron: Phenomenology and discovery potential in multijets, J. High Energy Phys. 07 (2008) 038.

[111] M. E. Peskin and D. V. Schroeder, An Introduction to Quantum Field Theory (Addison-Wesley, Reading, MA, 1995).

[112] J. M. Cline, G. Dupuis, and Z. Liu, LHC constraints on dark matter with $(130 \mathrm{GeV})$ gamma ray lines, J. High Energy Phys. 09 (2013) 065.

[113] W. Kwong, P. B. Mackenzie, R. Rosenfeld, and J. L. Rosner, Quarkonium annihilation rates, Phys. Rev. D 37, 3210 (1988).

[114] M. Aaboud et al. (ATLAS Collaboration), Search for new phenomena in dijet events using $37 \mathrm{fb}^{-1}$ of $p p$ collision data collected at $\sqrt{s}=13 \mathrm{TeV}$ with the ATLAS detector, Phys. Rev. D 96, 052004 (2017).

[115] CMS Collaboration, Report No. CMS-PAS-EXO-16-056.
[116] N. Craig, P. Draper, C. Kilic, and S. Thomas, Shedding light on diphoton resonances, Phys. Rev. D 93, 115023 (2016).

[117] M. Aaboud et al. (ATLAS Collaboration), Search for new phenomena in high-mass diphoton final states using $37 \mathrm{fb}^{-1}$ of proton-proton collisions collected at $\sqrt{s}=13 \mathrm{TeV}$ with the ATLAS detector, Phys. Lett. B 775, 105 (2017).

[118] M. Aaboud et al. (ATLAS Collaboration), Search for new high-mass phenomena in the dilepton final state using $36.1 \mathrm{fb}^{-1}$ of proton-proton collision data at $\sqrt{s}=13 \mathrm{TeV}$ with the ATLAS detector, J. High Energy Phys. 10 (2017) 182.

[119] M. Aaboud et al. (ATLAS Collaboration), Search for scalar leptoquarks in pp collisions at $\sqrt{s}=13 \mathrm{TeV}$ with the ATLAS experiment, New J. Phys. 18, 093016 (2016).

[120] CMS Collaboration, Report No. CMS-PAS-EXO-16-007.

[121] A. M. Sirunyan et al. (CMS Collaboration), Search for third-generation scalar leptoquarks and heavy right-handed neutrinos in final states with two tau leptons and two jets in proton-proton collisions at $\sqrt{s}=13 \mathrm{TeV}$, J. High Energy Phys. 07 (2017) 121.

[122] CMS Collaboration, Report No. CMS-PAS-EXO-16-009.

[123] J. Albrecht, F. Bernlochner, M. Kenzie, S. Reichert, D. Staub, and A. Tully, Future prospects for exploring present day anomalies in flavour physics measurements with Belle II and LHCb, arXiv:1709.10308.

[124] D. S. M. Alves, S. R. Behbahani, P. Schuster, and J. G. Wacker, The cosmology of composite inelastic dark matter, J. High Energy Phys. 06 (2010) 113.

[125] S. Raby, S. Dimopoulos, and L. Susskind, Tumbling gauge theories, Nucl. Phys. B169, 373 (1980).

[126] M. J. Teper, Glueball masses and other physical properties of $\mathrm{SU}(\mathrm{N})$ gauge theories in $D=(3+1)$ : A Review of lattice results for theorists, arXiv:hep-th/9812187.

[127] See the review of QCD in Ref. [103].

[128] M. Czakon, The Four-loop QCD beta-function and anomalous dimensions, Nucl. Phys. B710, 485 (2005).

[129] A. K. Rai, R. H. Parmar, and P. C. Vinodkumar, Masses and decay constants of heavy-light flavor mesons in a variational scheme, J. Phys. G 28, 2275 (2002). 